\title{
Modelling of flow around hexagonal and textured cylinders
}

\author{
H. Karampour ${ }^{1}$, Z. Wu' ${ }^{1}$, J. Lefebure ${ }^{2}$, D. Jeng ${ }^{1}$, B. Simpson ${ }^{1}$ \\ ${ }^{1}$ Griffith School of Engineering, Griffith University, Gold Coast, QLD 4222, Australia \\ ${ }^{2}$ ENSTA ParisTech, 32 Bd Victor, 75015 Paris, France
}

Key terms: Fluid mechanics; Maritime engineering; Offshore Engineering; Hydraulics \& hydrodynamics; Pipes \& pipelines

\begin{abstract}
The flow regime around a hexagonal polygon with low Reynolds numbers $R e<200$ is numerically investigated in two different orientations namely face-oriented and corneroriented. The basic flow characteristics, including drag coefficient, lift coefficient, Strouhal number and critical Reynolds number of the hexagonal cylinders, are calculated using 2D transient numerical analysis. Within the studied range of $R e$, the predicted lift coefficient and Strouhal number of the face-oriented hexagon were higher than those of the corneroriented hexagon. In contrast, the predicted drag coefficient and critical Reynolds number of the corner-oriented hexagon were greater than those of the face-oriented. Flow characteristics of a novel textured geometry are also studied using 3D transient analysis. The Strouhal number $S_{t}$ of the textured geometry was found to be in between the $S_{t}$ of both the hexagonal cylinders, and its lift coefficient is lower than that of the hexagonal cylinders.
\end{abstract}

\section{Introduction}

Understanding characteristics of the flow regime around a bluff body and resultant forces are of importance in various engineering applications where fluid-structure interaction has to be considered such as wind engineering and offshore engineering. Extensive studies have been performed on analysing the unsteady wake around circular and square cylinders, through numerical modelling (Braza et al. 1986; Gallardo et al. 2017; Meliga et al. 2016; Norberg 1993; Palei and Seifert 2007; Park et al. 1998; Sohankar et al. 1998b; Swaddiwudhipong et al. 2007; Travin et al. 2000; Baldock at al. 2014) and laboratory experiments (Bearman and Zdravkovich 1978; Norberg 1994; Sumner and Akosile 2003; Williamson 1989; Wood et al. 2016;

Zdravkovich 2003). However, in contrast, the flow regime behind polygonal cylinders has received only limited attention. The wake flow behind a circular cylinder firstly becomes unstable beyond a critical Reynolds number and then a periodic flow regime known as a Von Karman vortex street develops. The point of flow separation varies depending on the upstream flow parameters such as $\mathrm{Re}$, surface roughness etc. In case of flow past polygons, the flow detaches from the cylinder at the sharp corners of the leading or rear edges, forming the vortex regime on either side of symmetry plane of the polygon. Studying the behaviour of the vortices, the pressure and the friction distribution and in-line and cross-flow forces on the cylinder are of great practical importance because of their destructive effects such as fatigue failure caused by vortex induced vibration (VIV).

Sohankar et al. (1998a) investigated the flow regime around a square cylinder with $R e=200$. Their numerical simulations showed that the Strouhal number increased from 0.17 at angle of attack $\alpha=0^{\circ}$ (face orientation) to 0.20 at $\alpha=45^{\circ}$ (corner orientation). Yang et al. (2010) found the critical Reynolds number to be $\mathrm{Rec}=45$ and 40 for face-oriented and corner-oriented squares respectively. The dynamics of flow past hexagonal cylinders has received only limited attention so far. Sparrow et al. (2004) investigated the heat transfer from hexagonal cylinders and found 
that while all faces were engaged in a recirculation zone in case of face-orientation, only four faces with corner-orientation experienced flow separation. Tian and Wu (2009) studied the twodimensional flow around regular polygons with even number of edges at low Reynolds numbers. They found critical Reynolds numbers for vortex shedding in steady and unsteady flows. They derived Eq. (1) for Strouhal number of a polygon (StN) as a function of edge number of the polygon (N) and Strouhal number of a circle $(\mathrm{St} \infty)$ at $47<\mathrm{Re}<180$.

$$
\begin{aligned}
& S_{t N}=\Upsilon\left(0.2684-1.0356 \sqrt{\frac{\Upsilon}{\mathrm{Re}}}\right), \\
& S_{t \infty}=0.2684-1.0356 \sqrt{\frac{\Upsilon}{\mathrm{Re}}} \\
& \Upsilon=\frac{N}{\pi} \sin \left(\frac{N-1}{N} \pi\right) \Gamma\left(\frac{N-1}{N}\right)^{2} \Gamma\left(\frac{N+2}{N}\right)
\end{aligned}
$$

Khaledi and Andersson (2011) studied the vortex shedding of face-oriented and corner-oriented hexagons using transient three-dimensional models at $R e=100,500$ and 1000 . When comparing the wake regime between square and hexagonal cylinders at different orientations, they realised that wake dynamics is not influenced by the front stagnation point being positioned on a face or at a corner but rather if the width of the projected cylinder is determined by sharp corners or flow-parallel faces. Although the vortex regime and streamline patterns in the wake of hexagonal cylinders were investigated in their research, a thorough investigation of unsteady lift and drag forces, pressure and friction distributions and critical Reynolds numbers requires more attention.

This study aims to investigate the flow regime around hexagonal polygons in two different orientations namely the face-oriented and the corner-oriented at low Reynolds numbers. The present study is focused on low Reynolds numbers $(R e<200)$ where the laminar flow regime and Karman vortex street are expected regardless of the shape of the cylinder (Khaledi and Andersson 2011). It is well known that a transition from 2D to 3D flow occurs in the wake behind a circular cylinder when the Reynolds number is about 170 (Williamson 1988; Williamson 1996).

This results in a drop in the shedding frequency (St) whereas drag and lift are probably little affected. It is likely that this transition from $2 \mathrm{D}$ to $3 \mathrm{D}$ wake flow depends on the shape of the cross-section and it is also likely to guess that an earlier transition to three-dimensional flow will occur for non-spherical cross-sections. However, since the major concern of this study are drag and lift forces, the authors believe that usage of the present 2D results even at $\mathrm{Re}=200$ can be justified. Rajani et al. (2009) used the finite volume method and showed that for circular cylinder at $R e<200$ there is no significant difference between the hydrodynamic forces CD, CL and Strouhal number St computed from 2D and 3D analyses. Flow characteristics around a textured geometry which has a hexagonal cross-section that changes orientation from a face-oriented to a corner-oriented pattern along the length of the pipeline has been studied. Previous studies have shown superior performance of textured geometry over conventional cylindrical shapes with respect to major structural instability issues in subsea pipelines such as propagation buckling (Albermani et al. 2011; Stephan et al. 2015; Karampour et al. 2017) and buckle interaction (Karampour and Albermani 2014; Karampour and Albermani 2015). The vortex 
shedding mechanism of stationary hexagonal cylinders has been studied using transient twodimensional numerical simulations by common software. Forces in-line and normal to the uniform flow and corresponding pressure distributions on the hexagon faces have been calculated. Where possible the outcomes are compared against existing results reported in the literature. Finally, a three-dimensional transient fluid dynamics study of the vortex regime around a textured geometry is presented.

\section{Model description and validation}

Fig. 1a shows the rectangular fluid domain and boundary conditions. To do the comparison study, circular, square and hexagonal cylinders with dimensions shown in Fig. 1b were centred at equal distance of 30D from top and bottom symmetry boundaries. Zero normal velocity and stress were prescribed on the symmetry boundaries. The blockage ratio (ratio of diameter of equivalent circular cylinder $D$ to width of the domain) was taken as 0.017 (Linnick and Fasel 2005). Uniform velocity was assigned to the upstream boundary located at $30 \mathrm{D}$ from the centre of cylinder. The downstream boundary was located 60D away from the centre of the cylinder and was defined by zero stress component.

A finer mesh was adopted in the nearfield circular area around the cylinder with diameter 10D and in strips of width 24D in the upstream, wake region and cross-flow directions. The twodimensional, incompressible, Navier-Stokes equations in the Cartesian coordinate can be written as follows:

$$
\begin{aligned}
& \nabla \cdot(\stackrel{1}{\prime})=0 \\
& \frac{\partial}{\partial t}(\stackrel{\mathrm{r}}{\rho v})+\nabla \cdot \underset{(\rho v v)}{\mathrm{rr}}=-\nabla p+\nabla \cdot(\tau)+\stackrel{\mathrm{r}}{F} \\
& \tau=\mu\left(\nabla v+\nabla v^{\mathrm{r}}\right)
\end{aligned}
$$

where $\mathrm{p}$ is the static pressure, $\mathrm{T}$ is the stress tensor, and $\mathrm{F}$ is the external body force. For the two-dimensional, incompressible, Navier-Stokes equations. The governing Eq. (2) is solved using semi-implicit SIMPLE algorithm with double precision accuracy in FLUENT solver (ANSYS 16.1 Release). In this algorithm the velocity correction is solved explicitly, while the momentum equation and pressure correction equations are solved implicitly. Time step of $0.01 \mathrm{sec}$ is adopted in order to resolve 50 vortex-shedding cycles (the accuracy is justified based on results of Fig. 2 and Table 1). The total flow time required for the convergence of a numerical solution varies with Reynolds number and shape of the cylinders. For lower Reynolds numbers $(\operatorname{Re}<60)$, larger total flow times were required in order to attain desirable convergence.

\section{Table 2}

Frequencies of lift force, darg force and vortex shedding of Hexagonal cylinder, $R e=$ 200

\begin{tabular}{llll}
\hline & $\begin{array}{l}f_{L}(\mathrm{~Hz}) \\
\text { (frequency of lift force) }\end{array}$ & $\begin{array}{l}f_{D}(\mathrm{~Hz}) \\
\text { (frequency of drag } \\
\text { force) }\end{array}$ & $\begin{array}{l}f_{S}(\mathrm{~Hz}) \\
\text { (vortex shedding } \\
\text { frequency) }\end{array}$ \\
Corner-oriented & 0.175 & 0.353 & 0.175 \\
Face-oriented & 0.206 & 0.417 & 0.207 \\
\hline
\end{tabular}




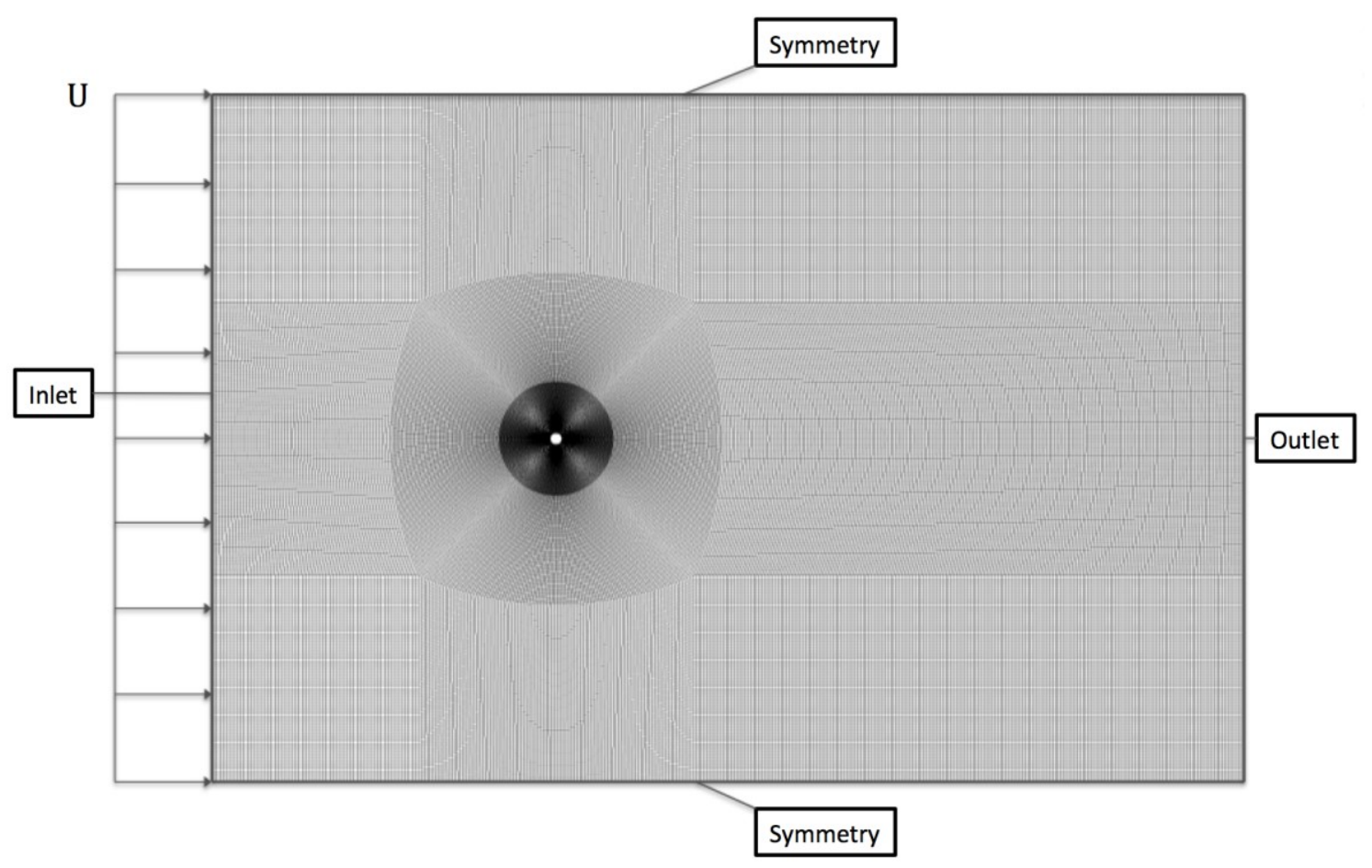

a
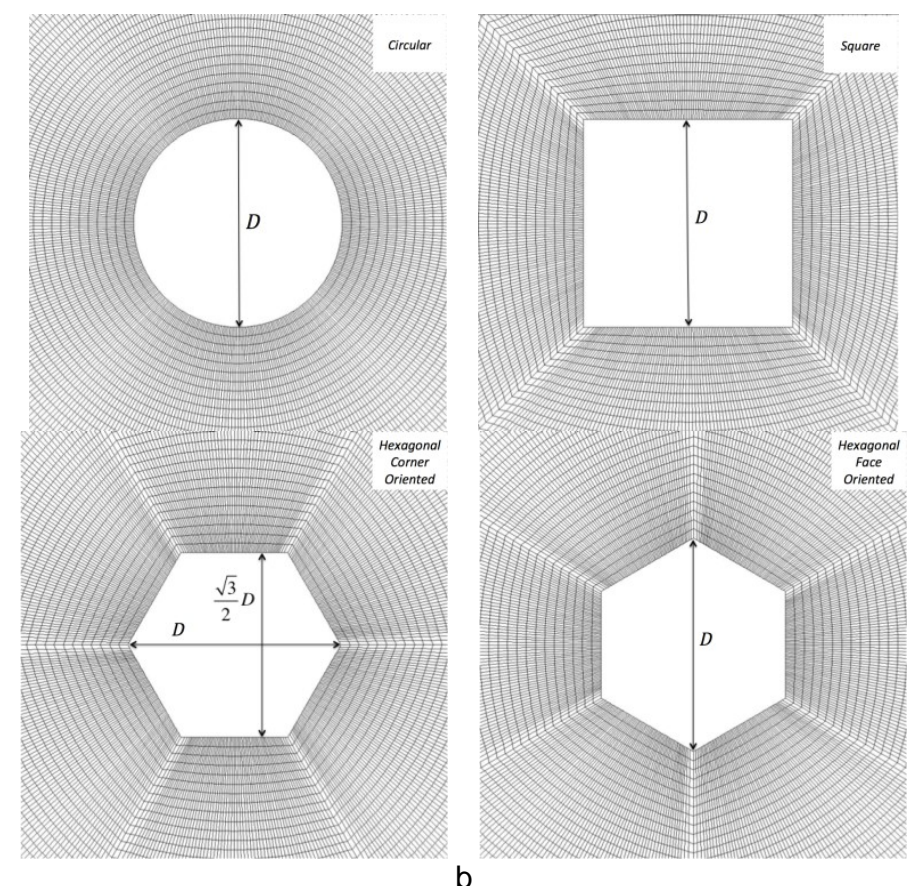

Figure 1: Geometry and mesh size (a) domain and boundary conditions and (b) studied cross sections.

A mesh sensitivity study is conducted on the circular shape at $R e=100$ and hydrodynamic coefficients are represented in Table 1. The hydrodynamic coefficients obtained from the present study with mesh 3 are in close agreement with the mean drag and the maximum lift coefficients reported in (Berthelsen and Faltinsen 2008; Linnick and Fasel 2005), which is based on an immersed boundary method. In addition, the Strouhal number calculated in the present 
study agrees well with the experimental value reported by Williamson and Roshko (1988). Using mesh 3, the mean drag coefficients for the square shape at low Reynolds numbers $(20<R e<200)$ are plotted in Fig. 2. The current CD values agree well with those obtained from the Direct Numerical Simulation presented by Okajima et al. (1997) and are consistently more accurate than the results reported by Singh and Biswas (2013) using a Finite Element Method (FEM). It should be noted that Singh and Biswas (2013) used a blockage ratio of 0.05 with 31,200 quadrilateral elements in their model, whereas in the current study blockage ratio of 0.017 with 134,160 quadrilateral elements are utilized. Furthermore, as will be discussed in the next section, the critical Reynolds numbers and pressure and friction coefficients obtained for circular and square cylinders using mesh 3 agree well with those reported in the previous studies. Therefore mesh 3 is preferred to mesh 4 and 5 (Table 1) due to its computational efficiency and is used hereafter in this study.

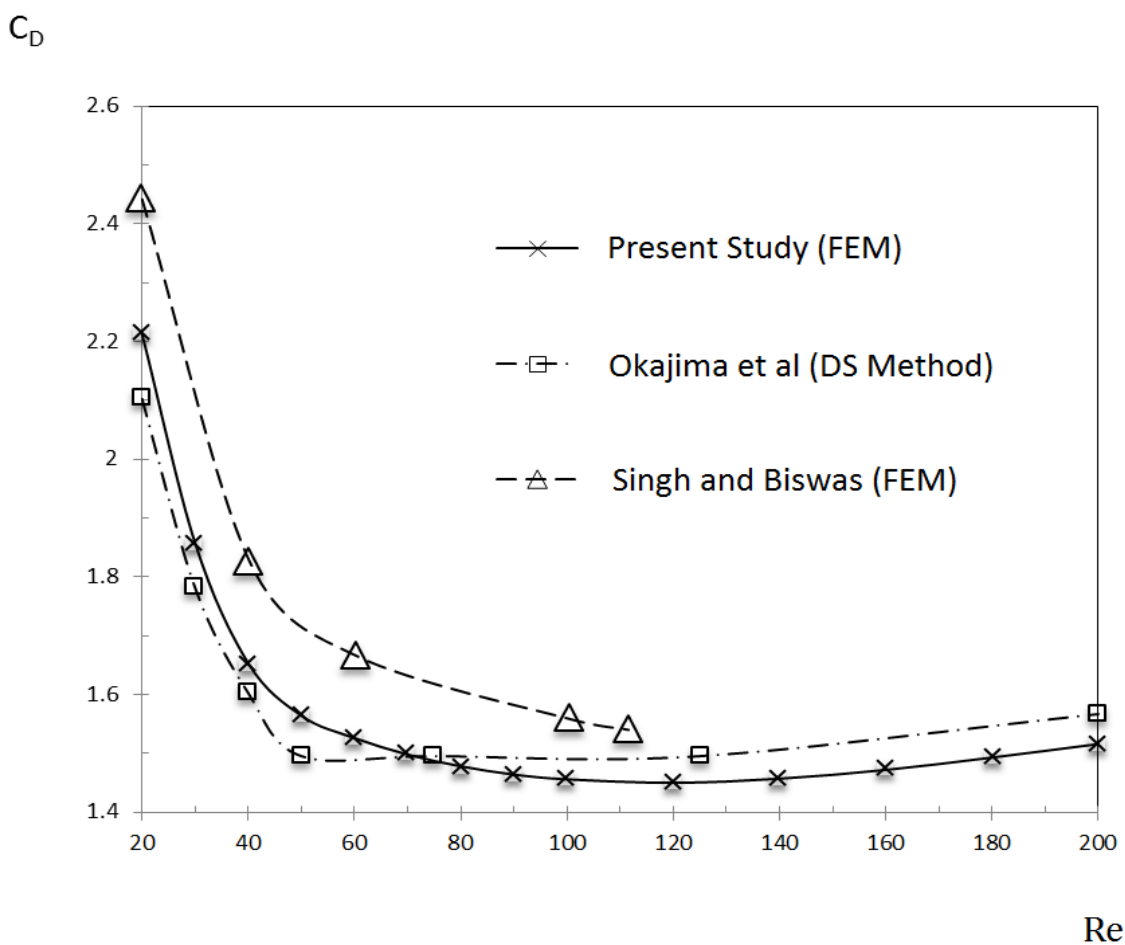

Figure 2: Variation of $C D$ as a function of $R e$, for square shape from different studies.

Table 1: Mesh sensitivity study showing Strouhal number, mean drag and max lift coefficients of Circular Cylinder, $R e=100$

\begin{tabular}{lcccc}
\cline { 2 - 5 } & \# of cells & $S_{t}$ & $C_{D}$ (mean drag) & $C_{L}$ (max lift) \\
\hline Linnick \& Fasel (2005) & - & 0.166 & 1.340 & \pm 0.333 \\
Berthelsen and Faltinsen (2008) & - & 0.169 & 1.380 & \pm 0.340 \\
Williamson and Roshko(1988) & - & 0.164 & - & - \\
The present study (mesh 1) & 33,900 & 0.158 & 1.394 & \pm 0.366 \\
The present study (mesh 2) & 76,425 & 0.161 & 1.381 & \pm 0.347 \\
The present study (mesh 3) & 134,160 & 0.163 & 1.363 & \pm 0.339 \\
The present study (mesh 4) & 212,625 & 0.163 & 1.352 & \pm 0.333 \\
The present study (mesh 5) & 306,300 & 0.163 & 1.348 & \pm 0.330 \\
\hline
\end{tabular}




\section{Numerical results and discussion}

\subsection{Unsteady lift and drag forces in laminar vortex regime}

Fig. 3 illustrates lift coefficient CL versus non-dimensional time for a corner-oriented hexagon at $R e=53,54$ and 55. The mean $C L$ is negligible (of the order of 10-4) but different growth/decay trends are observed at investigated Reynolds numbers. At $\mathrm{Re}=53$ and 54, the CL peaks appear to be decaying with time; while at $R e=55$ the peak values of lift coefficient show a gradual growth. The Fast Fourier Transformations (FFT) of the lift coefficients on the frequency domain (shown in Fig. 3) indicate an abrupt growth in amplitude of CL from order of 10-9 at $R e=54$ to order of $10-8$ at $R e=55$. Knowing that near the onset of wake instability the growth rate of $C L$ is close to zero, (Kumar and Mittal (2006)), one can deduct that the critical Reynolds number of a corner-oriented hexagon is between 54 and 55 and probably closer to $R e=54$. This critical Reynolds number is larger than the $\mathrm{Rec}=49$ reported in (Tian and Wu 2009) for a corneroriented hexagon which was derived from the equation of a stream function in a circular domain. However, it should be noted that for a circular cylinder, Tian and Wu (2009) found the critical Reynolds number to be 45 which is smaller than the $R e c=49$ reported by (Rajani,Kandasamy and Majumdar 2009) using implicit pressure based finite volume method. In the present study $\mathrm{Rec}=50$ was calculated for the circular cylinder which is quite close to the value reported by Rajani et al. (2009). In order to find the critical Reynolds number of a face-oriented hexagon, similar analysis was performed and time-variant lift results are shown in Fig. 4. At $\mathrm{Re}=51, \mathrm{CL}$ decays rapidly to almost zero while at $R e=52$ the decay rate is slower and non-zero $C L$ peak value is observed. With respect to the amplitude of time-variant lift from FFT (shown in Fig. 4) it can be deducted that the critical Reynolds number for a face-oriented hexagon must be around 52.

Mean drag coefficients CD, lift coefficients CL and Strouhal number St of cylinders shown in Fig. $1 \mathrm{~b}$ are plotted against Reynolds number in Fig. 5a. While interpreting results in Fig. 5, it should be noted that for a corner-oriented hexagon according to Fig. 1b, a characteristic diameter of $0.87 \mathrm{D}$ is adopted whereas for a face-oriented hexagon the characteristic diameter is $\mathrm{D}$. The CD results shown in Fig. 5a raises several interesting points: (1) Within the studied range of Reynolds numbers, the corner-oriented hexagon shows higher drag coefficient compared to the face-oriented hexagon, however this trend is predicted to be reversed for Re $>200$ by extrapolating current results, (2) The face-oriented hexagon shows smaller CD compared to that of a circular cylinder up to the Reynolds number of 130 and (3)The CD of a square cylinder and corner-oriented hexagon are almost identical up to $R e=160$. The mean drag coefficients of faceoriented hexagon and square cylinders exhibit rapid growth for $R e>130$, while the corneroriented hexagon shows almost constant drag coefficient $(C D=1.45)$ beyond $R e=130$. 

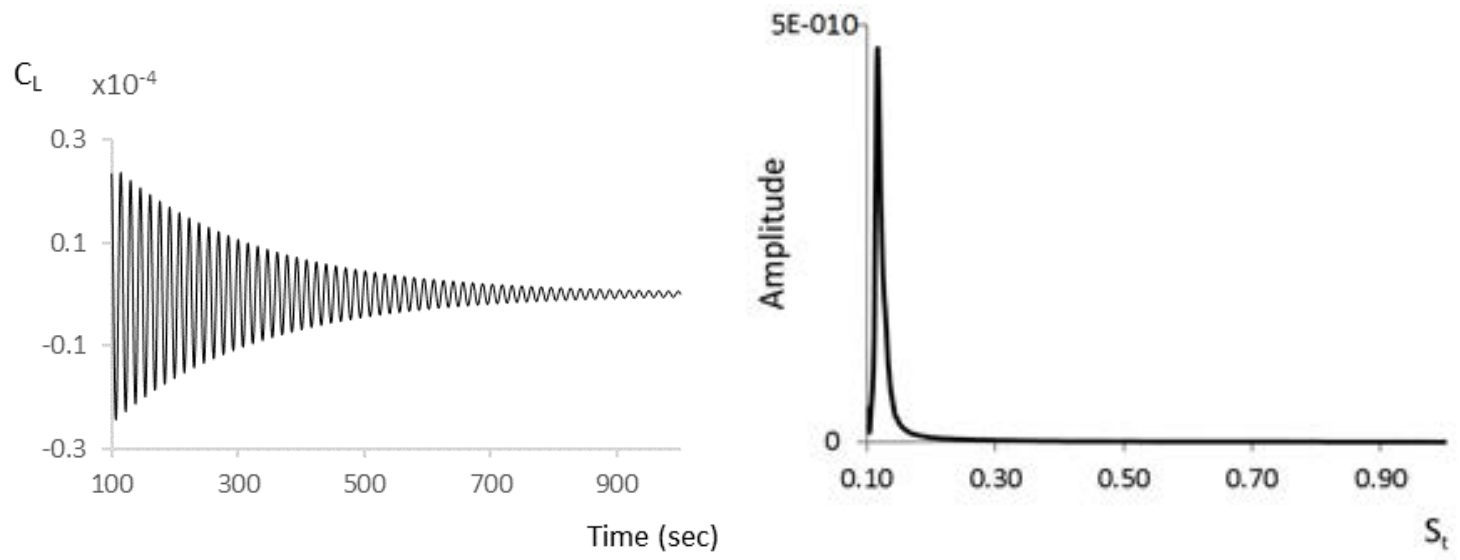

$R e=53$
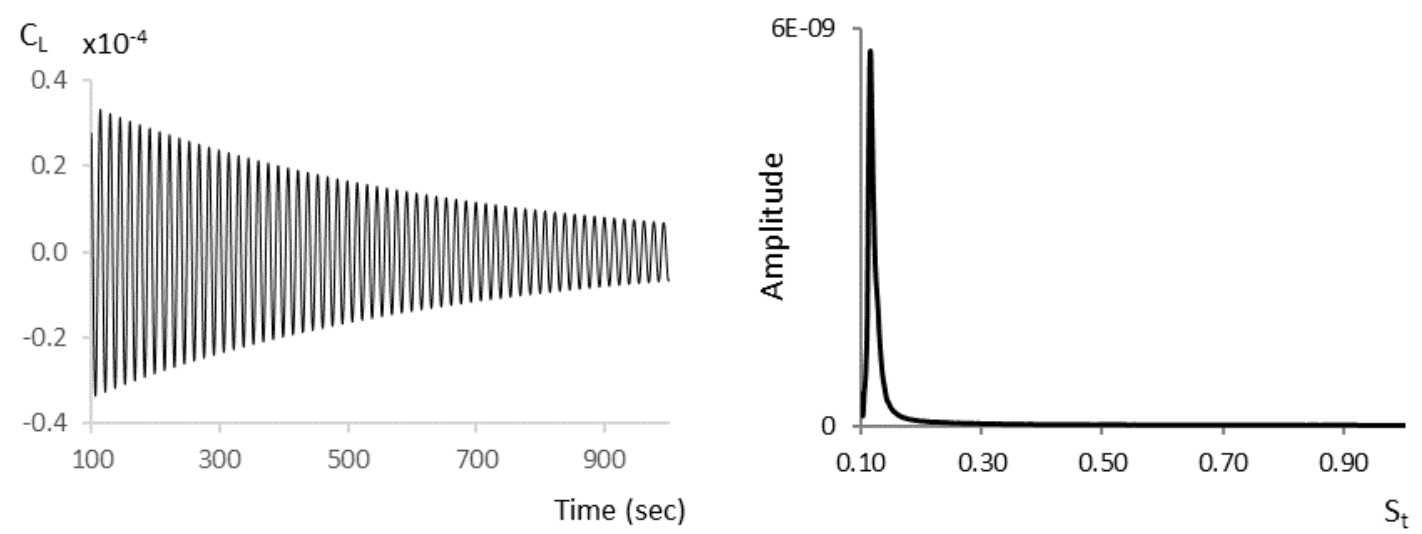

$R e=54$
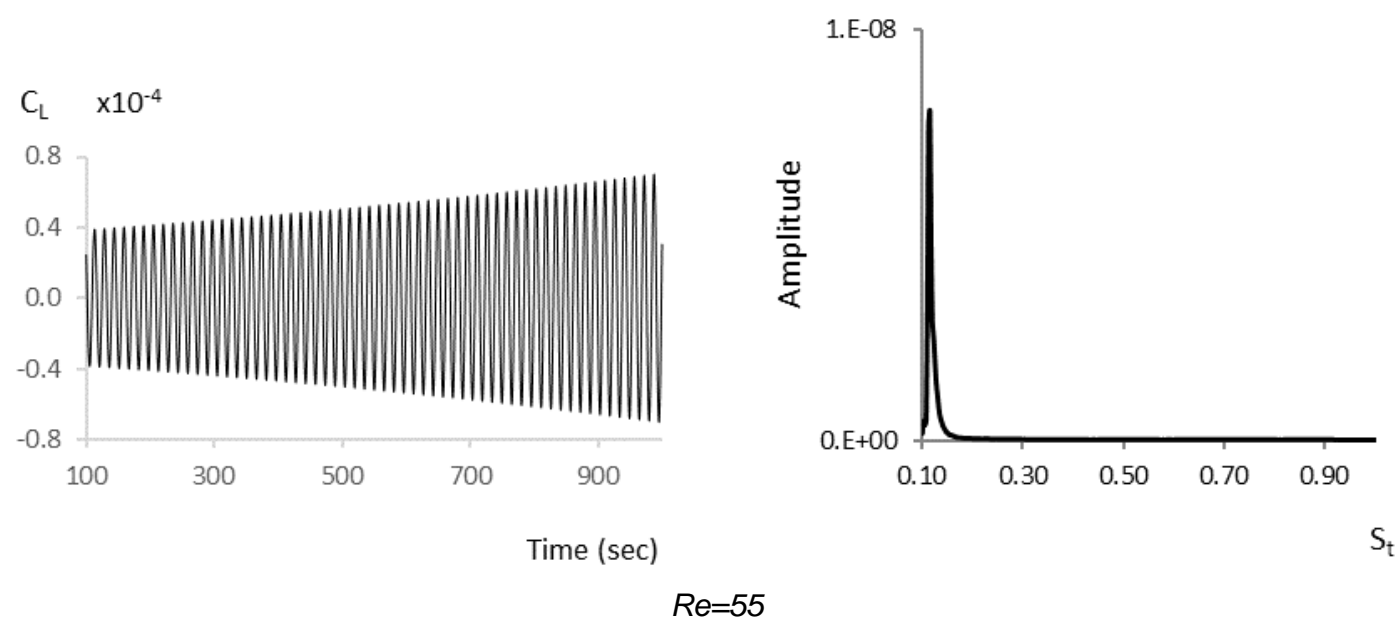

Figure 3: Lift time history and amplitude of corner-oriented hexagon at $\mathrm{Re}=53,54$ and 55. 
$\mathrm{C}_{\mathrm{L}} \times 10^{-4}$

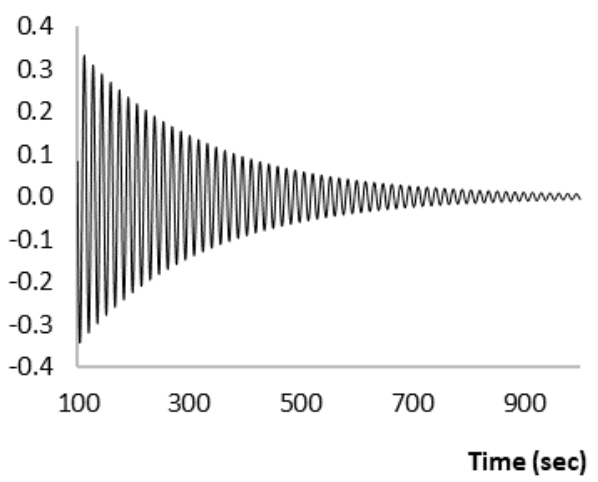

$R e=51$

$C_{L}$

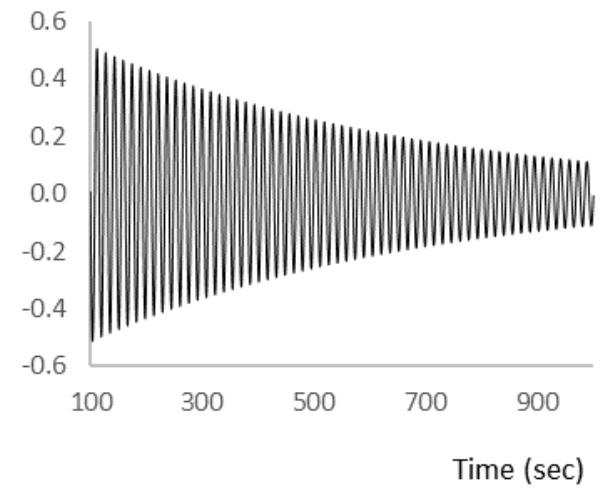

$C_{L}$

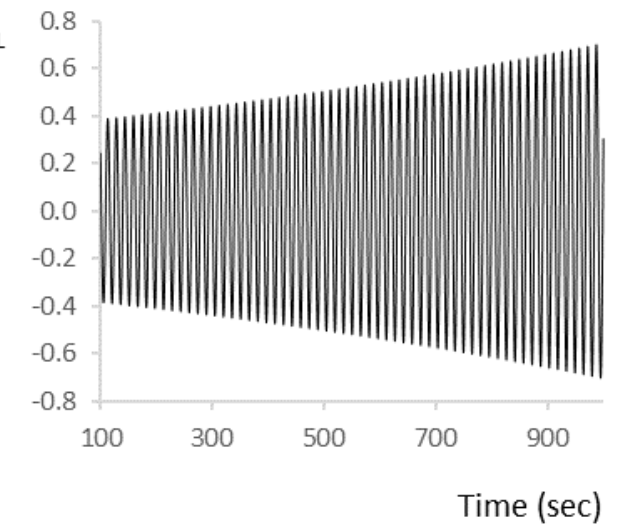

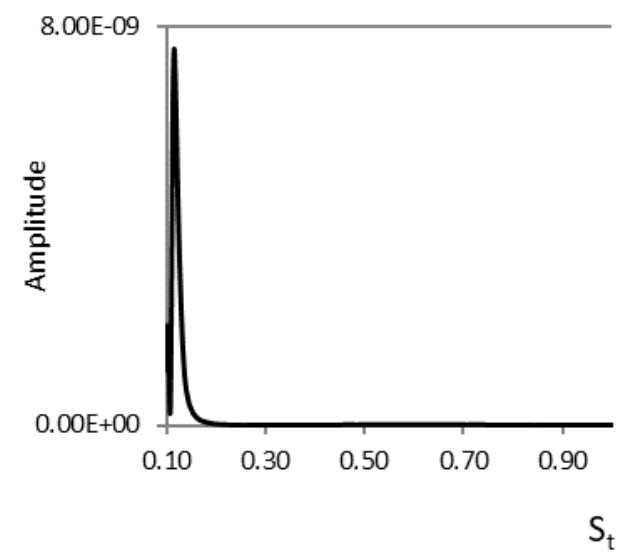

$\mathrm{S}_{\mathrm{t}}$

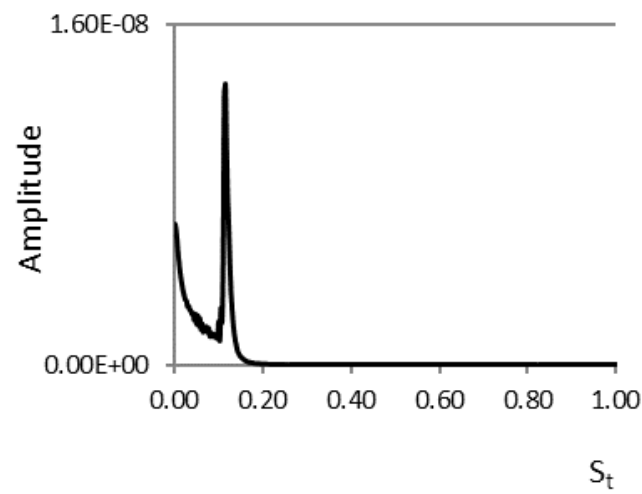

$R e=52$

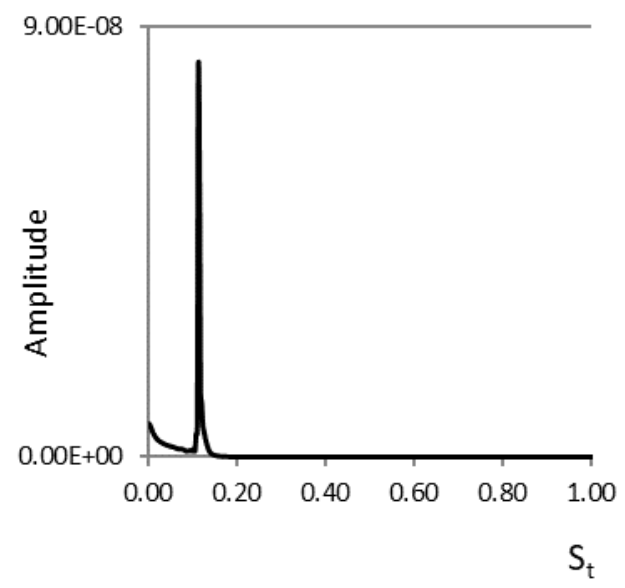

$R e=53$

Figure 4: Lift time history and amplitude of face-oriented hexagon at Re=51, 52 and 53. 


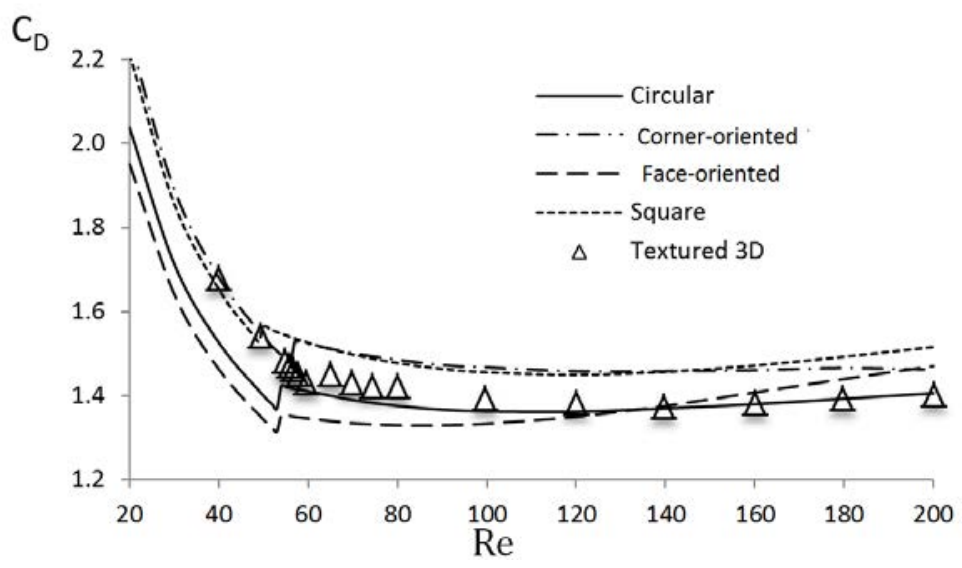

a

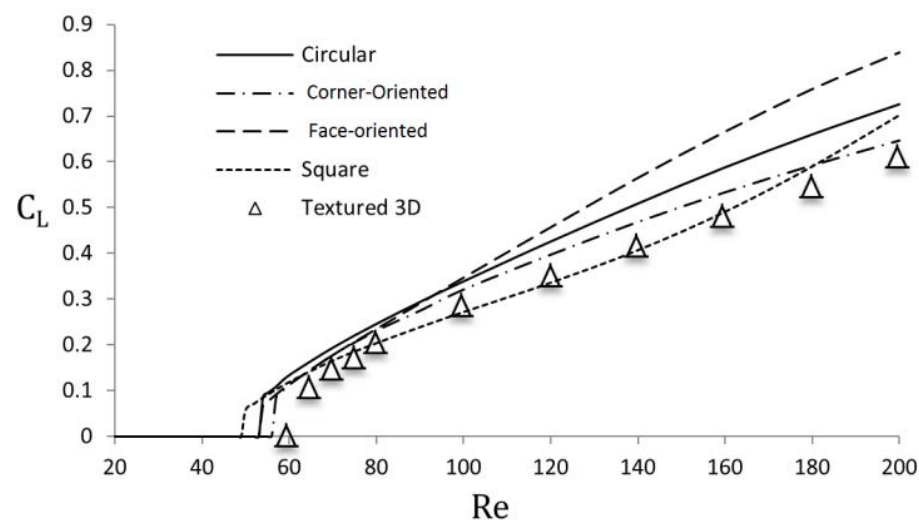

b

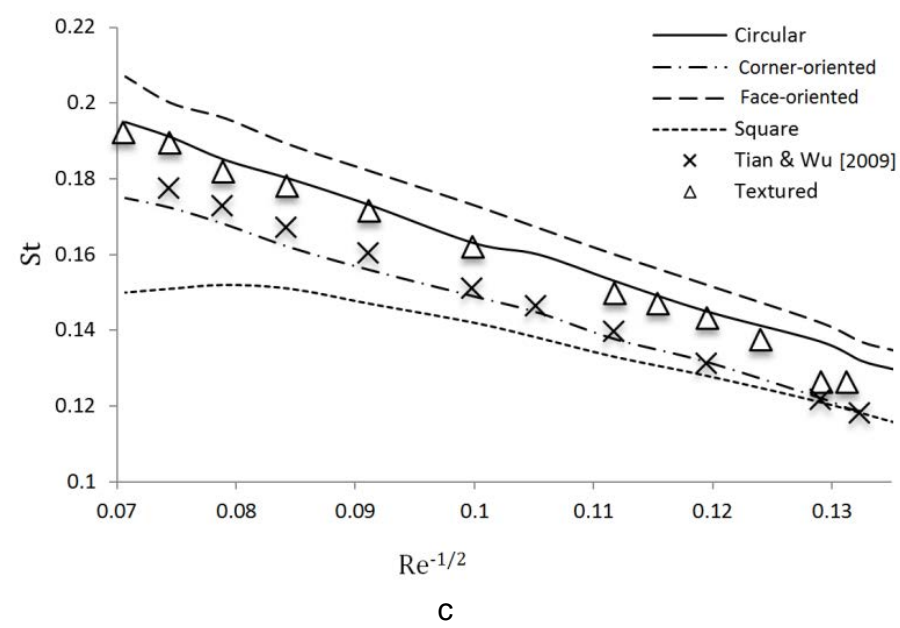

Figure 5: Mean drag coefficient CD, maximum lift coefficient $C L$ and Strouhal number St of studied cases.

The kinks observed in the drag curves for Reynolds numbers between 40 and 60 correspond to the corresponding critical Reynolds numbers. The peak lift coefficients $C L$ are represented in Fig. 5b. The hexagonal and circular cylinders show analogous CL up to a Reynolds number equal to 80 . For Re>80, the lift coefficient of the corner-oriented hexagon plunges while the 
face-oriented hexagon shows a monotonically increasing CL. As expected, the lift coefficient of the circular cylinder is between those of corner-oriented and face-oriented hexagons for Reynolds numbers between 100 and 200. The square cylinder exhibits the lowest CL up to $R e=180$. Strouhal numbers for various cylinders are plotted against inverse of square root of Reynolds number ( ) in Fig. 5c. Strouhal number St is defined in Eq. (3), where fs is the vortex shedding frequency, $U$ is the stream velocity and $D$ is the characteristic diameter shown in Fig. $1 b$.

$$
S_{t}=\frac{f_{s} D}{U}
$$

The St results presented here are in good agreement with those reported for a corner-oriented hexagon by Tian and Wu (2009). Unlike other geometries, the square cylinder does not show a linear reduction of $\mathrm{St}$ versus $\mathrm{Re}-1 / 2$. The maximum Strouhal number of the square shape shown in Fig. $5 c$ corresponds to shedding frequency $f s=0.152$ at Reynolds number of 160 . Frequencies of inline force (fD) and cross-flow force (fL) and vortex shedding frequency (fs) of hexagonal cylinders at $R e=200$ are shown in Table 2 . Regardless of the orientation of the hexagonal cylinder, the lift force oscillates at the vortex shedding frequency fs, while the drag force oscillates at frequency $2 \mathrm{fs}$. The same relationship between the lift and drag forces and shedding frequencies are valid in stationary circular cylinders (Sumer and Fredsøe 1997).

Table 2: Frequencies of lift force, drag force and vortex shedding of Hexagonal cylinder, $R e=200$

\begin{tabular}{llll}
\hline & $f_{L}(\mathrm{~Hz})$ & $f_{D}(\mathrm{~Hz})$ & $f_{S}(\mathrm{~Hz})$ \\
& (frequency of lift force) & $\begin{array}{l}\text { (frequency of drag } \\
\text { force) }\end{array}$ & $\begin{array}{l}\text { (vortex shedding } \\
\text { frequency) }\end{array}$ \\
Corner-oriented & 0.175 & 0.353 & 0.175 \\
Face-oriented & 0.206 & 0.417 & 0.207 \\
\hline
\end{tabular}

\subsection{Pressure and friction distribution}

Time-averaged surface pressure coefficients (Cp, defined in Eq. (4)) at Reynolds numbers of 100 and 200 are depicted in Fig. $6 a$, and b respectively for a circular cylinder as well as the face-oriented and the corner-oriented hexagons.

$$
C_{p}=\frac{p}{\frac{1}{2} \rho U^{2}}
$$

In Eq. (4), $p$ is the surface pressure and $\rho$ is the density of fluid. Due to symmetry, surface pressures for half-cross sections are plotted in Fig. 6 , where $\theta=0^{\circ}$ corresponds to the stagnation point on the front face and $\theta=180^{\circ}$ corresponds to the base point on the extreme rear end of the cylinder. In all cases, pressures at the rear side of the cylinders are negative and remain practically constant, which can be related to weaker flow in the wake region as compared to that of the front region. Pressure coefficients in the front and in the wake region of the circular cylinder at $R e=100$ found in the present study are in excellent agreement with experimental measurements of Homann (1936). However at $R e=200$ the obtained negative pressure coefficients in the wake region are lower than those reported in (Thom 1933). Rajani et al. (2009) found similar discrepancies between their results and Thom's (1933) experimental 
measurements and related it to the possible three-dimensional regime of the wake observed in Thom's experiments. Circular, corner-oriented and face-oriented hexagons show almost identical stagnation pressure $\mathrm{Cp} 0$ (pressure at $\theta=0^{\circ}$ ) and mean base pressure $\mathrm{Cpb}$ (pressure at $\theta=180^{\circ}$ ) at $\mathrm{Re}=100$. However, the base pressure of face-oriented hexagon $\mathrm{Cpb}=-1.14$ is $18 \%$ higher than those of circular and corner-oriented cylinders at $R e=200$. With respect to the timeaveraged pressure forces, the corner-oriented hexagon has the highest negative pressure among the cylinders.

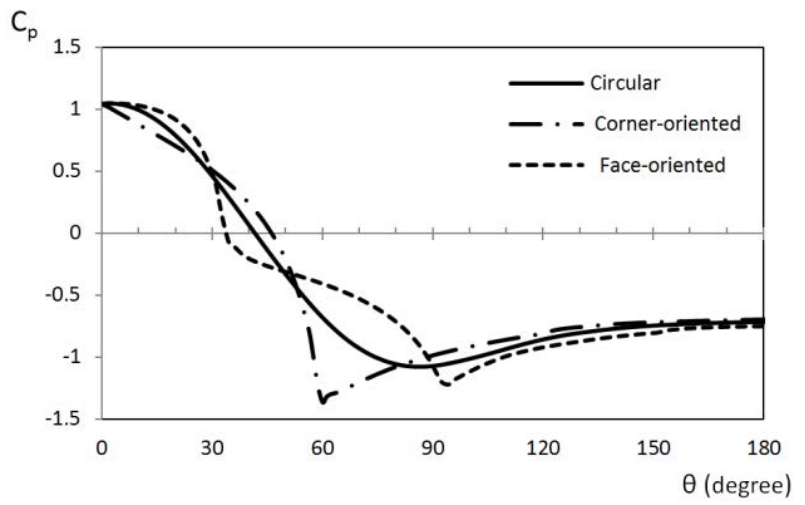

a

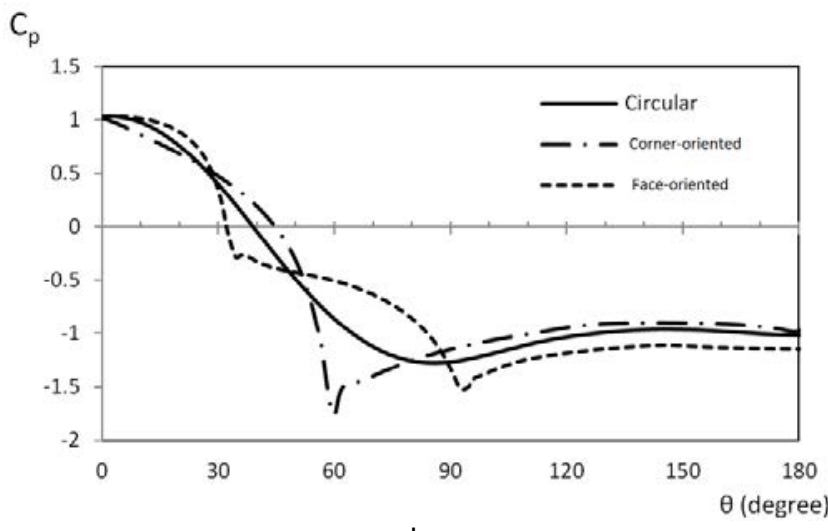

b

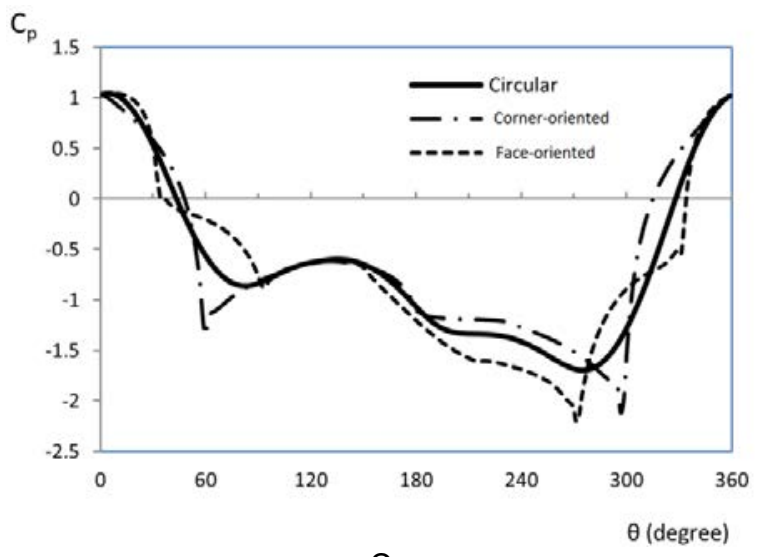

C

Figure 6: Pressure distribution on the surface of hexagonal cylinders, (a) time-averaged at $\mathrm{Re}=100$, (b) time-averaged at $R e=200$ and (c) onset of maximum negative lift force at $R e=200$.

Fig. $6 \mathrm{c}$ shows that the peak suction pressure $\mathrm{Cpb}=-2.25$ occurs at maximum negative lift force in the face-oriented hexagon at Reynolds number of 200. 


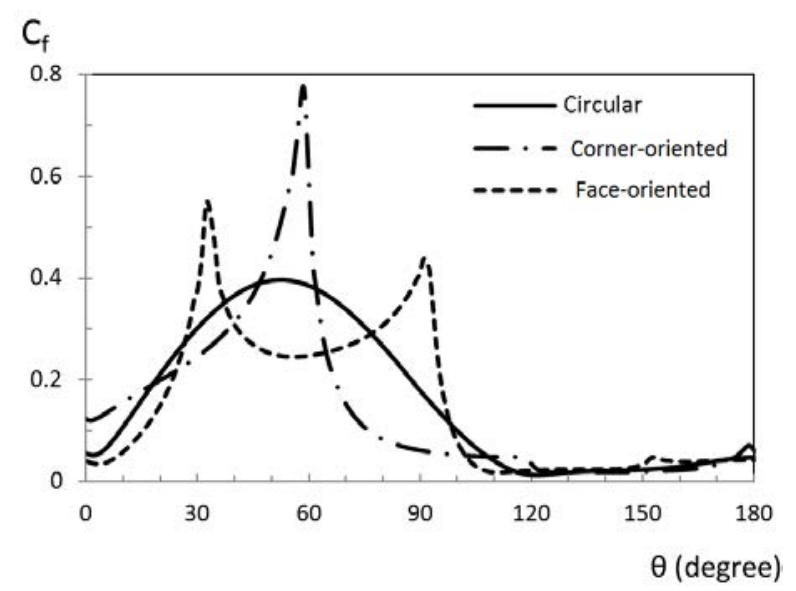

a

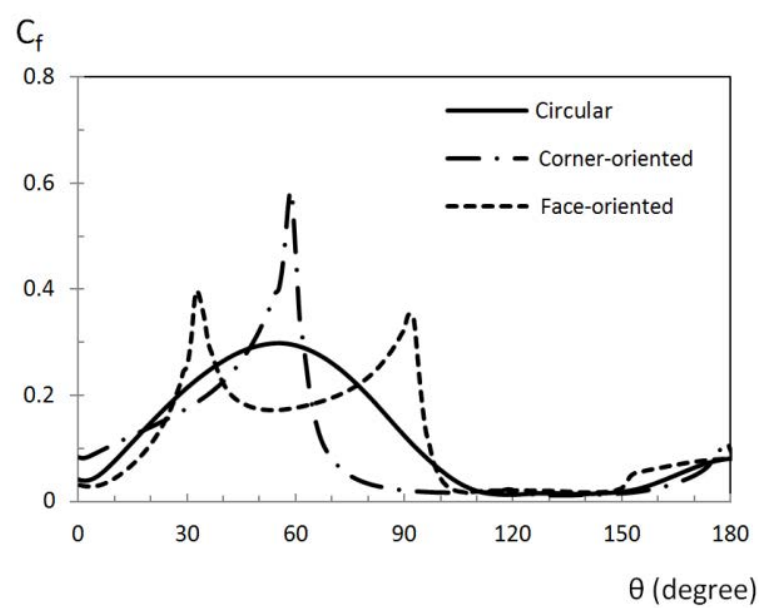

b

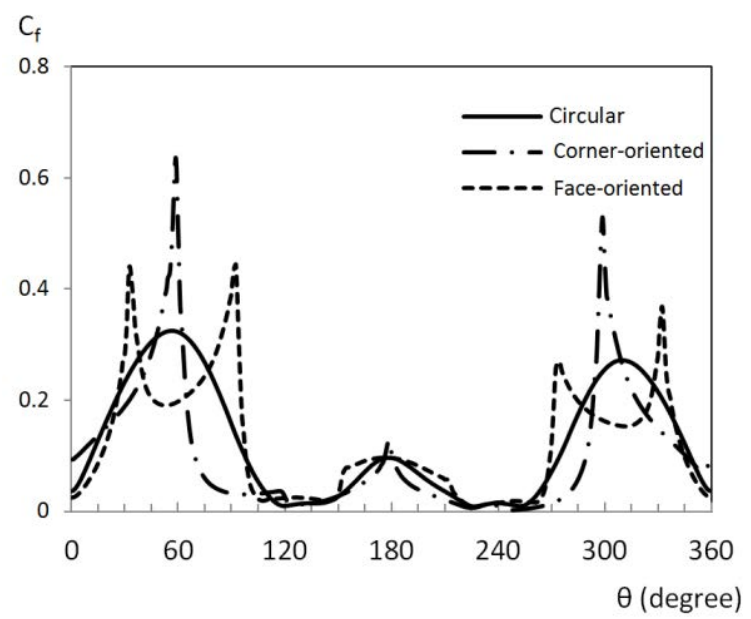

C

Figure 7: Skin friction on the surface of hexagonal cylinders, (a) time-averaged at $\mathrm{Re}=100$, (b) timeaveraged at $R e=200$ and (c) onset of maximum negative lift force at $R e=200$.

Figure 7 shows absolute values of time-averaged surface friction coefficients $\mathrm{Cf}$. Surface friction coefficient is related to the wall shear stress $\mathrm{T}$ as shown in Eq. (5). 


$$
C_{f}=\frac{\tau}{\frac{1}{2} \rho U^{2}}
$$

The friction forces decrease with the increase in Reynolds number from 100 to 200 which is due to the decrease in the boundary layer thickness (Sumer and Fredsøe 1997). As shown in Figs. $7 a$ and $b$, the peak value of $C f$ occurs at the front edge of hexagonal cylinders (i.e. $\theta=60^{\circ}$ in corner-oriented hexagon and $\theta=30^{\circ}$ in face-oriented hexagon) and is substantially higher than that of circular cylinder. The friction coefficients corresponding to the onset of maximum lift for $\mathrm{Re}=200$ are shown in Fig. 7c. It is evident that the maximum friction forces occur on the upstream side of the cylinders. Unlike pressure coefficients, peak friction coefficients at maximum lift are not much higher than time-averaged friction coefficients. Comparison between Figs. $6 \mathrm{c}$ and $7 \mathrm{c}$ at $\mathrm{Re}=200$ shows that peak absolute $\mathrm{Cp}$ of the corner-oriented hexagon is almost equal to that of the face-oriented hexagon. However, peak Cf value of the corneroriented hexagon is $42 \%$ higher than that of the face-oriented hexagon. It is worth noting that the current peak friction coefficients for a circular cylinder of $\mathrm{Cf}=0.4$ and $\mathrm{Cf}=0.3$ at Reynolds numbers of 100 and 200, respectively; match the results of Rajani et al. (2009) but are smaller than the experimental results of Dimopoulos and Hanratty (1968). Rajani et al. (2009) associated the discrepancies to the imprecise prediction of the separation point in the experiments.

\subsection{Streamline patterns and spanwise vorticities}

In order to study the behaviour of spanwise vortices, the time development of the shedding process in the near-wake region is illustrated in Figs. 8 and 10 for the face-oriented and the corner-oriented hexagons at $\mathrm{Re}=200$. Four snapshots of the vortex regime are presented, with each figure separated by $\mathrm{Ts} / 4$ seconds. This represents one shedding cycle from the respective geometries. In Figs. 8 and 10, panel (a) corresponds to the onset of the shedding cycle i.e. a zero lift coefficient with negative slope on the $C L$ versus time curve and panels $(b-d)$ correspond to the minimum lift, zero lift with positive slope and maximum lift, respectively. Since the unit inlet velocity $(U)$ and the unit characteristic diameter $(D)$ were assumed in the finite volume model, the period of shedding cycle, Ts, is equal to the Strouhal number St. 


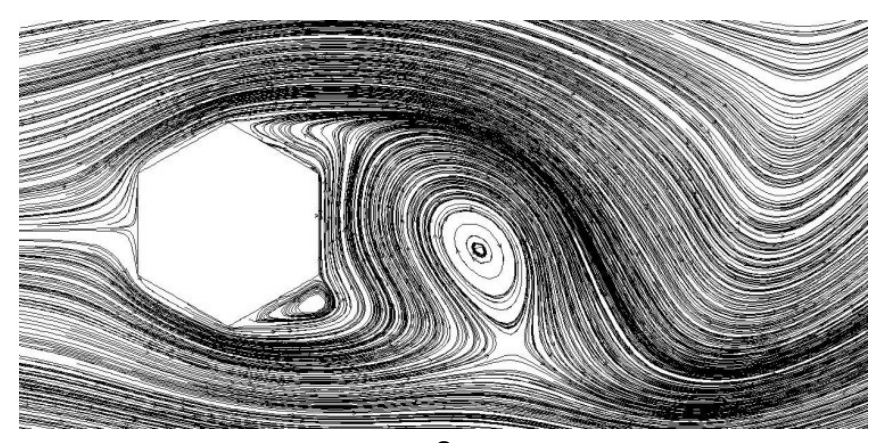

a

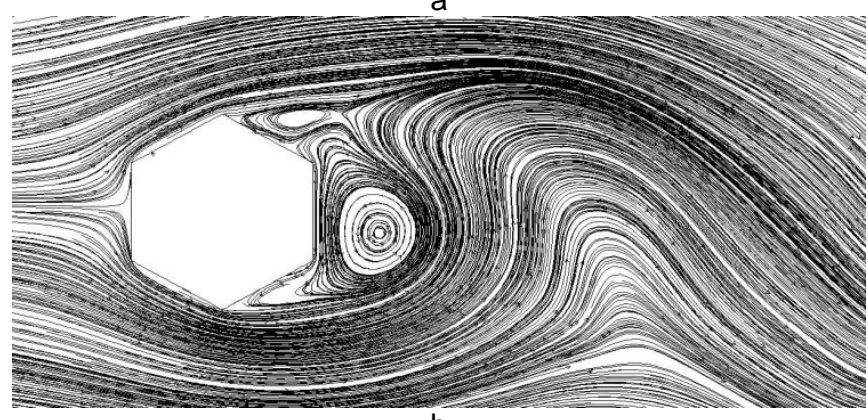

b

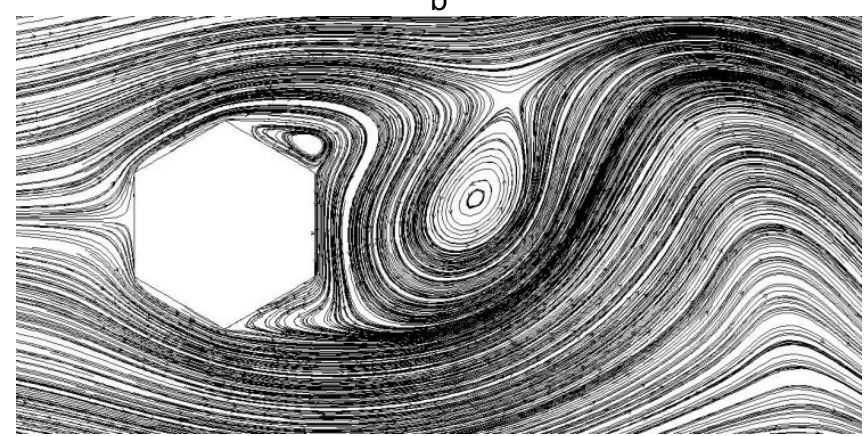

C

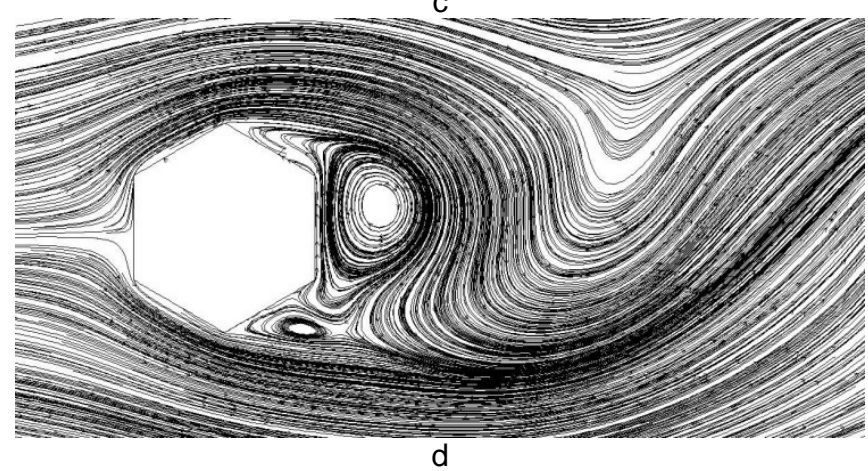

Figure 8: Streamlines of flow around the face-oriented hexagon at $R e=200$ (a) $C L=0$ with negative slope, (b) $C L=\operatorname{Min},(c) C L=0$ with positive slope and (d) $C L=$ Max. 


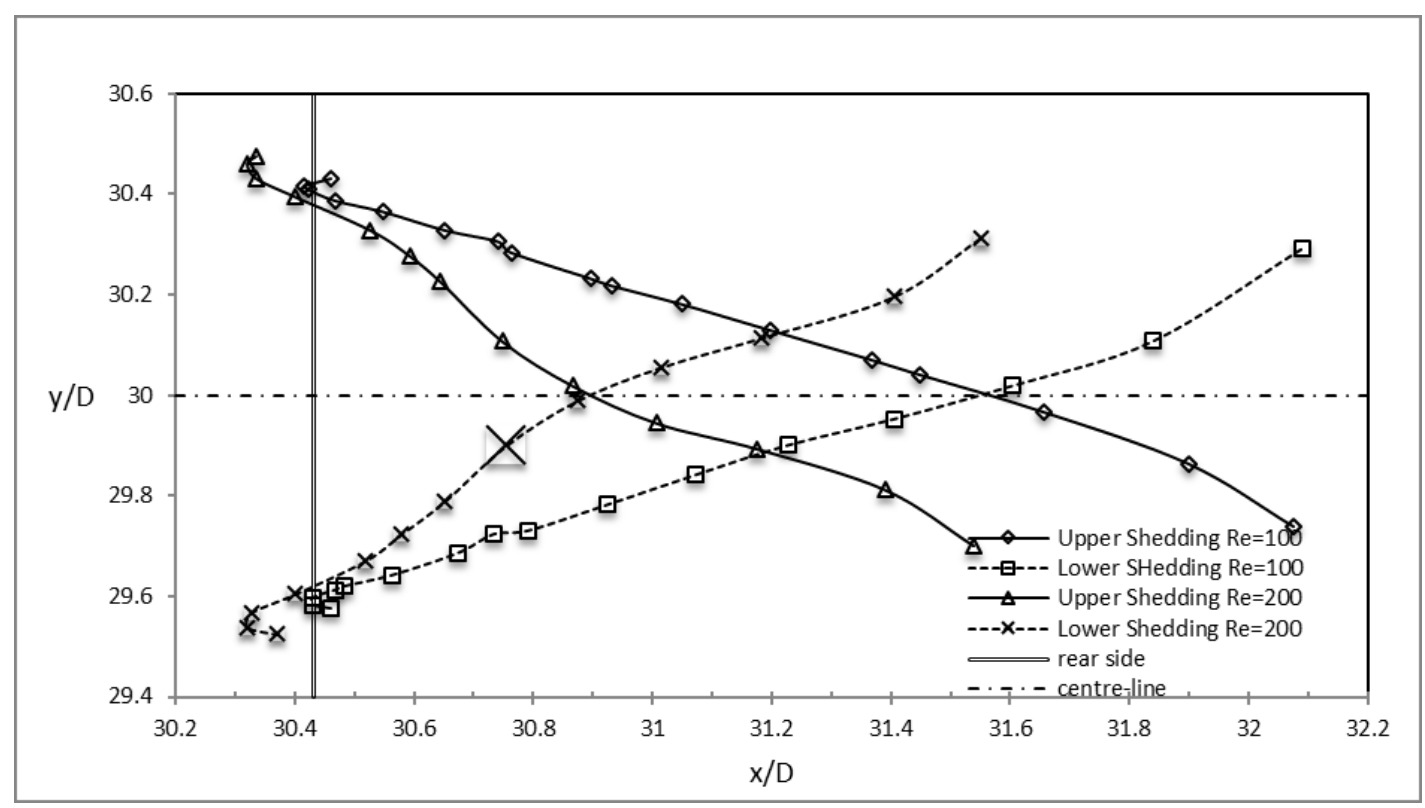

Figure 9: Trajectory of vortex centres in the near-wake region of face-oriented hexagon at $\mathrm{Re}=100$ and $\mathrm{Re}=200$.

As shown in Fig. 8 the unsteady near-wake flow regime downstream of the face-oriented hexagon is comprised of a counter-clockwise vortex, a clockwise vortex and a saddle-point which is develops due to the intersection of the streamlines. At the beginning of the shedding cycle (Fig. 8a), a counter-clockwise vortex is shed behind the lower sharp edge and is countered by a larger clockwise vortex in the rear wake region. As the counter-clockwise vortex grows, it moves towards the centre back of the hexagon's wall. At the same time, a new clockwise vortex is developing on the upper edge of the hexagon. At minimum lift (Fig. 8b), the counter-clockwise vortex is located closely behind the hexagon face and is spread between the upper and lower sides of the hexagon. This vortex is centred $0.34 \mathrm{D}$ downstream and $0.12 \mathrm{D}$ below the centre of the hexagon face. The coordinates of the centre point are highlighted in the trajectory plot of the lower shedding vortex $(R e=200)$ in Fig. 9. The streamline patterns for the positive lift force depicted in Figs. $8 \mathrm{c}$ and $8 \mathrm{~d}$ are almost mirror-images of negative lift force patterns in Figs. 8a and 8b. Unlike the clear saddle points observed for zero lift forces, no saddle points are seen at the maximum and minimum lifts. The coordinates of the centre of the lower (counter-clockwise) and upper (clockwise) vortices downstream of the face-oriented hexagon are shown in Fig. 9 for Reynolds numbers of 100 and 200. The data points correspond to different times within a cycle. Clockwise and counter-clockwise vortices are developed upstream of the hexagon's rear side and move towards the centre-line of the hexagon as time lapses. At $R e=100$, the upper and lower vortices intersect at a distance of 1.14D downstream of the hexagon's rear side. This value is close to the 1.1D reported by Khaledi and Andersson (2011). It is evident from Fig. 9 that vortices at $R e=200$ are located closer to the hexagon wall compared to vortices at $\mathrm{Re}=100$. The proximity of the vortex to the hexagon wall contributes towards the asymmetry of pressure distribution over the hexagon's surface. This explains the higher lift forces observed at $\mathrm{Re}=200$ compared to those of $\mathrm{Re}=100$ (almost twice as large as shown in Fig. 5b). 

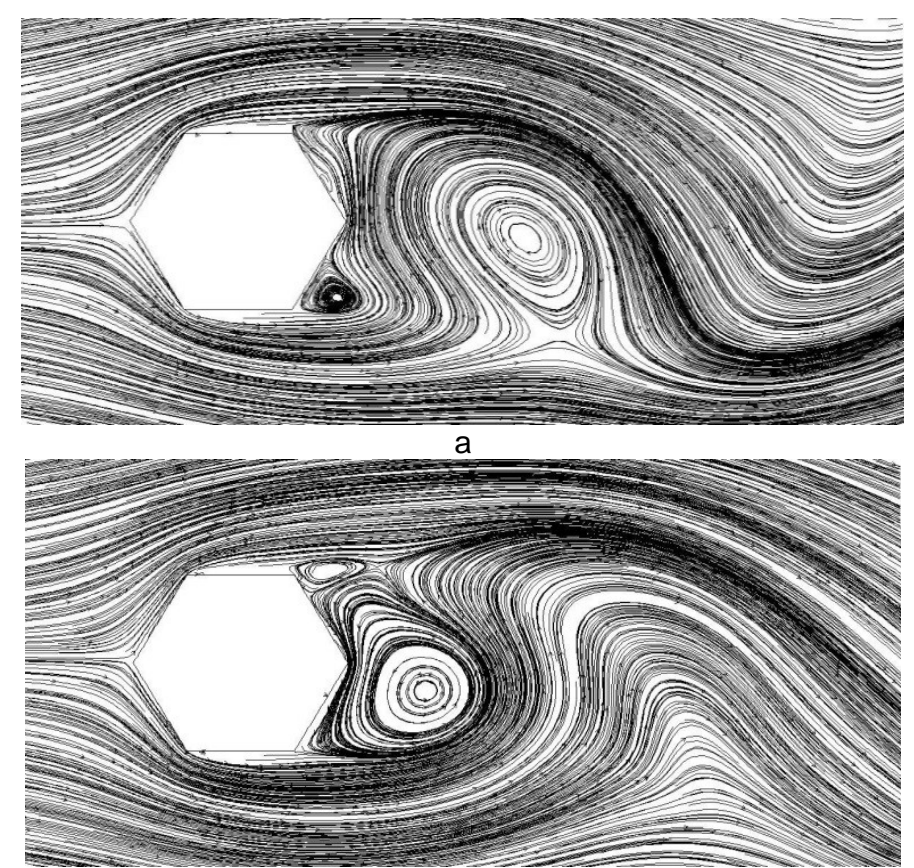

b

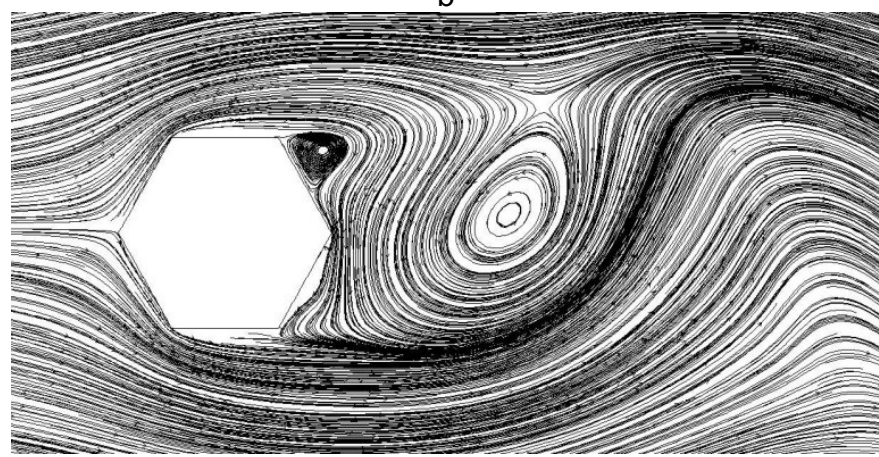

C

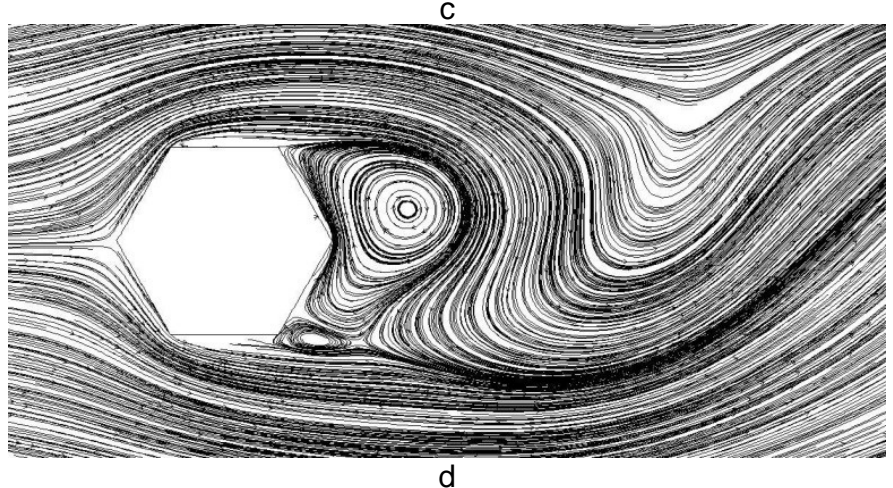

d

Figure 10: Streamlines of flow around the corner-oriented hexagon at $\mathrm{Re}=200$ (a) $\mathrm{CL}=0$ with negative slope, (b) $C L=M i n$, (c) $C L=0$ with positive slope and (d) $C L=M a x$. 


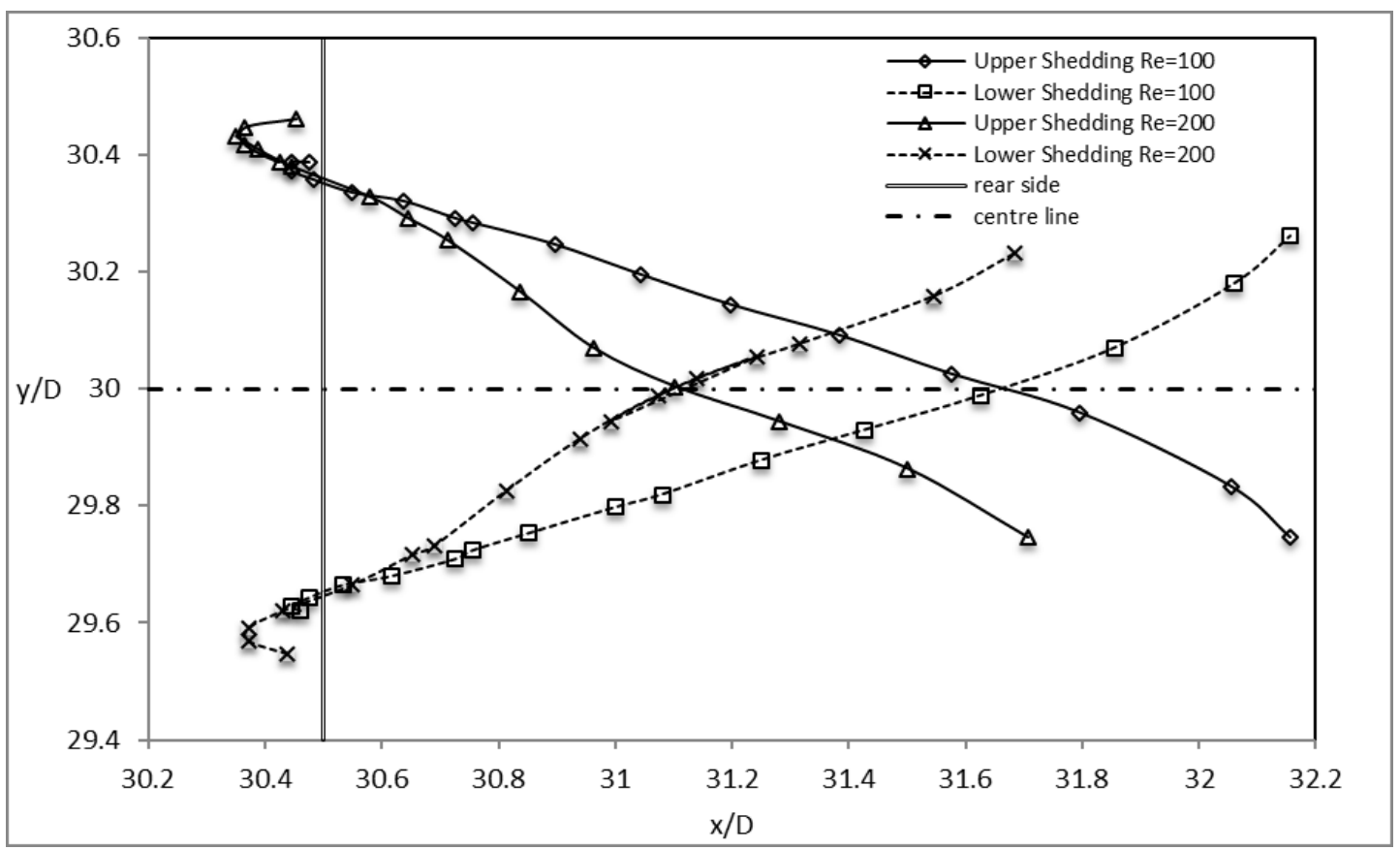

Figure 11: Trajectory of vortex centres in the near-wake region of corner-oriented hexagon at $\mathrm{Re}=100$ and $\mathrm{Re}=200$

The streamline of the corner-oriented hexagon at $R e=200$ is shown in Fig. 10. Two vortices, a counter-clockwise one at the lower edge and a simultaneous clockwise vortex $0.85 \mathrm{D}$ farther downstream of the hexagon wall are seen at zero lift in Fig. 10a. The fully-grown counterclockwise vortex approaches the cylinder wall a quarter of cycle later at minimum lift as shown in Fig. 10b. Comparison between streamline patterns at minimum lift in Figs. $8 \mathrm{~b}$ and 10b shows that the counter-clockwise vortex in the face-oriented hexagon covers a wider surface area around the cylinder compared to those of the corner-oriented hexagon. This surface area relates to the area corresponding to negative $\mathrm{CP}$ in the pressure distribution shown in Fig. 6c and is evidently wider in face-oriented hexagons than in corner-oriented hexagons. The trajectory of vortex centres for the corner-oriented hexagon is depicted in Fig. 11. The upper and lower vortices at $R e=200$ intersect $0.6 \mathrm{D}$ to the rear of the hexagon wall which is farther away than the intersection point $0.44 \mathrm{D}$ calculated for the face-oriented hexagon. Similar analysis was performed for other geometries. The results showed that at $R e=100$, the intersection points are located at 1.2D $1.7 \mathrm{D}, 1.15 \mathrm{D}$ and 1.14D for the circular, square, corneroriented and face-oriented shapes, respectively. With all the shapes, the vortices at $R e=200$ are centred closer to the pipe wall compared to those of $R e=100$.

\subsection{Application: Textured geometry}

Albermani et al. (2011) proposed a new pipeline geometry (textured geometry) that has the potential to increase the resilience of pipeline to propagation buckling and buckle interaction without the associated increase in wall-thickness. A textured pipe with a linearized diamond pattern was proposed as shown in Fig. 12. The textured geometry is an isometric replacement of the conventional circular pipe (also shown in Fig. 12) with the same material, diameter and wall-thickness. The basic building block of the diamond pattern is the isosceles triangular facet. 

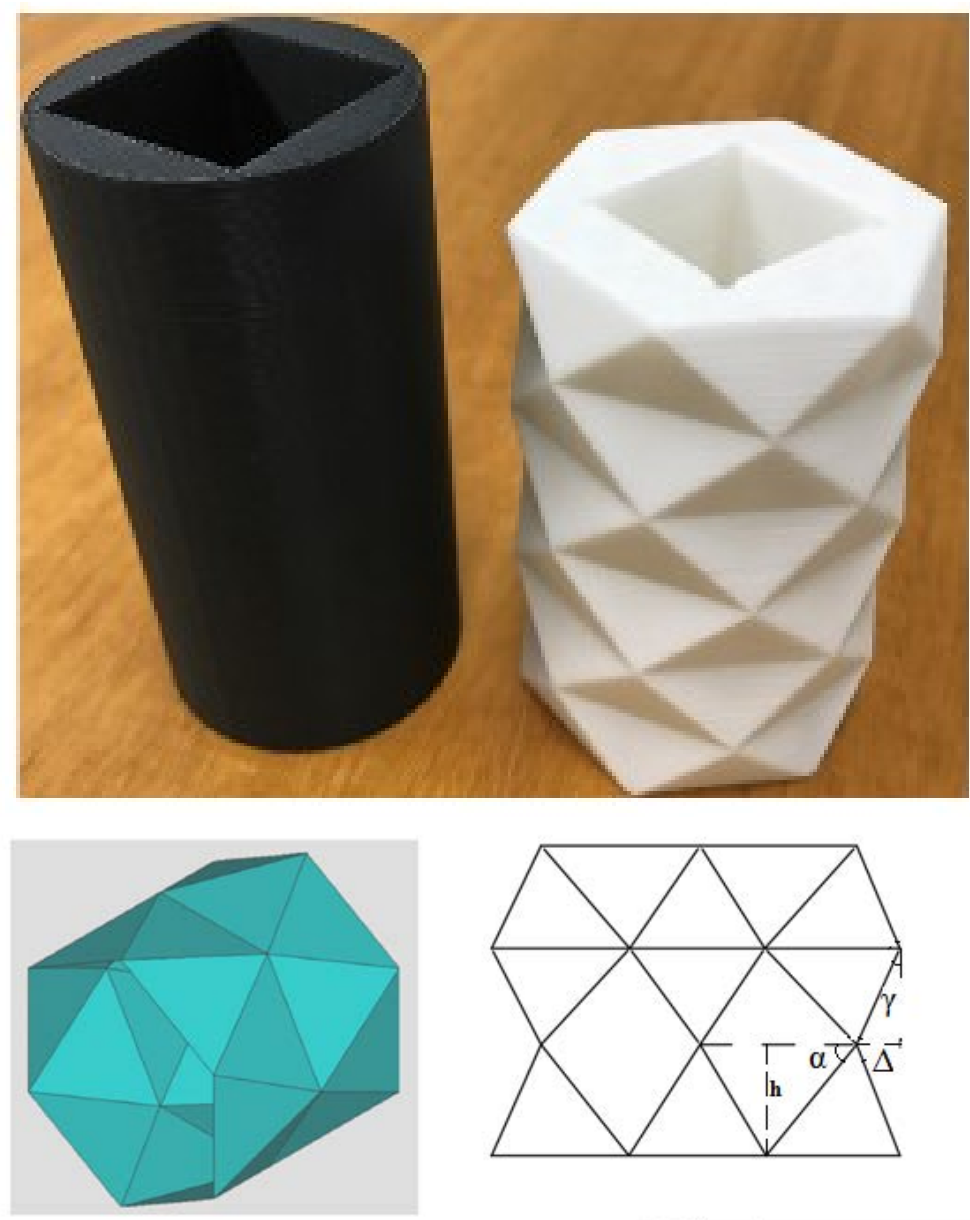

$$
\begin{aligned}
& \theta=\frac{\pi}{2}-\frac{\pi}{N} \\
& \lambda=2 R \sin \frac{\pi}{N} \\
& \Delta=R\left(1-\cos \frac{\pi}{N}\right) \\
& h=R \sin \frac{\pi}{N} \tan \alpha \\
& \gamma=\sin ^{-1} \frac{1-\cos \frac{\pi}{N}}{\sin \frac{\pi}{N} \tan \alpha}
\end{aligned}
$$

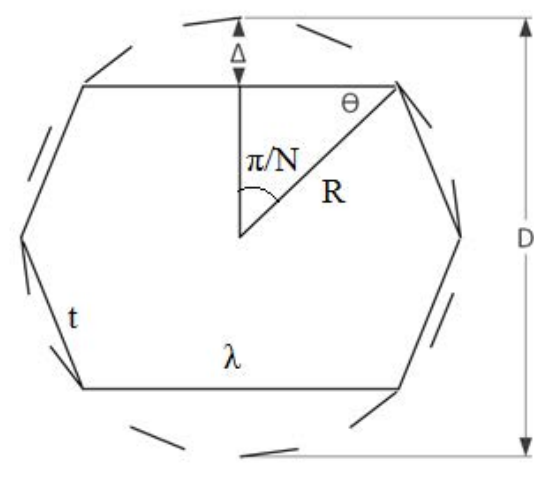

Figure 12: Circular, textured pipe and geometric parameters for a textured pipe.

The geometry of the textured pipe can be described in terms of four basic parameters as shown in Fig. 12. These are; the radius of the equivalent cylinder, $\mathrm{R}$; the wall-thickness, tw; the number of circumferential triangles, $N$, and the base angle of a typical triangular facet, $\alpha$. For the textured pipe shown in Fig. 12, $N=6$ and the remaining five geometric parameters $(\theta, \lambda, \Delta, \mathrm{h}$ and $\mathrm{y}$ ) are functions of $\mathrm{R}, \mathrm{N}$ and $\alpha$ as shown in Fig. 12. As a case study, $\mathrm{N}=6$ and $\alpha=450$ are assumed. As shown in Fig. 12, the cross section of the textured geometry comprises of a corner-oriented hexagon and a face-oriented hexagon distance $h$ apart. Three-dimensional transient analysis was performed where a pipe with a length of 20D was modelled. The width and length of the domain (Fig. 1) was reduced from 60D and 90D to 30D and 45D respectively, and the 3D-mesh was created by extruding the 2D-mesh. Initially 3D circular pipe models were created at $R e=100$ and $R e=200$. Flow regime characteristics such as the drag coefficient, lift 
coefficient and Strouhal number calculated from the 3D models were the same as those calculated from 2D models and reported in the experimental results. After performing a mesh sensitivity analysis, a mesh with over 2 million cells and seven hundred thousand nodes was adopted for the textured pipe. It has to be mentioned that the textured pipe does not maintain a constant characteristic length along its length. The characteristic length of the textured geometry (the dimension facing the flow) changes from 1.0D for the face-orientation to $0.87 \mathrm{D}$ for the corner-orientation. Therefore in the discussion that follows the characteristic length of the textured pipe will be assumed to be the mean of these values i.e. $0.93 \mathrm{D}$.

The time-history of the lift coefficient as well as the power spectra at $\mathrm{Re}=57,58$ and 59 are displayed in Fig. 13. The time history of lift coefficient at $R e=59$ shows a rapid growing trend which indicates that the critical Reynolds number has already been reached. The decaying trend in lift force observed at $\mathrm{Re}=57$ and 58 suggests that the critical Reynolds number has to be between those Reynolds numbers. The power spectra over frequency domain for those Reynolds (Fig. 13), also indicates that the critical Reynolds number of the textured geometry is between $\mathrm{Re}=57$ and 58 and closer to $\mathrm{Re}=57$. The critical Reynolds number found for the textured pipe is therefore higher than those of all the other studied shapes. The drag coefficient, lift coefficient and Strouhal number of the textured geometry are shown in Fig. 5 for Reynolds numbers up to 200 . For $\operatorname{Re}<50$, the drag coefficient of the textured pipe is larger than that of the circular pipe and closer to the CD of the square cylinder. The drag coefficient of the textured pipe rapidly becomes lower than that of the square cylinder at $50<R e<100$ and almost equal to that of the circular cylinder at Re $>100$. The drag coefficient of the textured pipe is consistently lower than that of the corner-oriented hexagon over the studied Re range and lower than CD of the face-oriented hexagon at $R e>130$. Interestingly, the lift coefficient of the textured pipe, shown in Fig. 5b, is smaller than CL of all the other shapes within the studied Re range. For Reynolds numbers up to 160 , the lift coefficient of the textured geometry is almost the same as that of the square cylinder, but drastically drops at higher Re values. The Strouhal number of the textured geometry is between the St of the face-oriented and corner-oriented hexagons, and becomes closer to that of the circular cylinder for higher Reynolds numbers (smaller Re-1/2 shown in Fig. 5c).

The wavy geometry of the textured shape can be defined by two non-dimensional parameters: the normalised amplitude $\Delta / D$ and the normalised wave-length $h / D$, where (D-2. $\Delta$ ) is the minimum diameter shown in Fig. 12. Lam et al. (2004) defined a non-dimensional parameter to express the degrees of cylinder obliqueness:

$$
c o=\frac{\Delta^{2}}{0.5 h(D-2 \Delta)}
$$




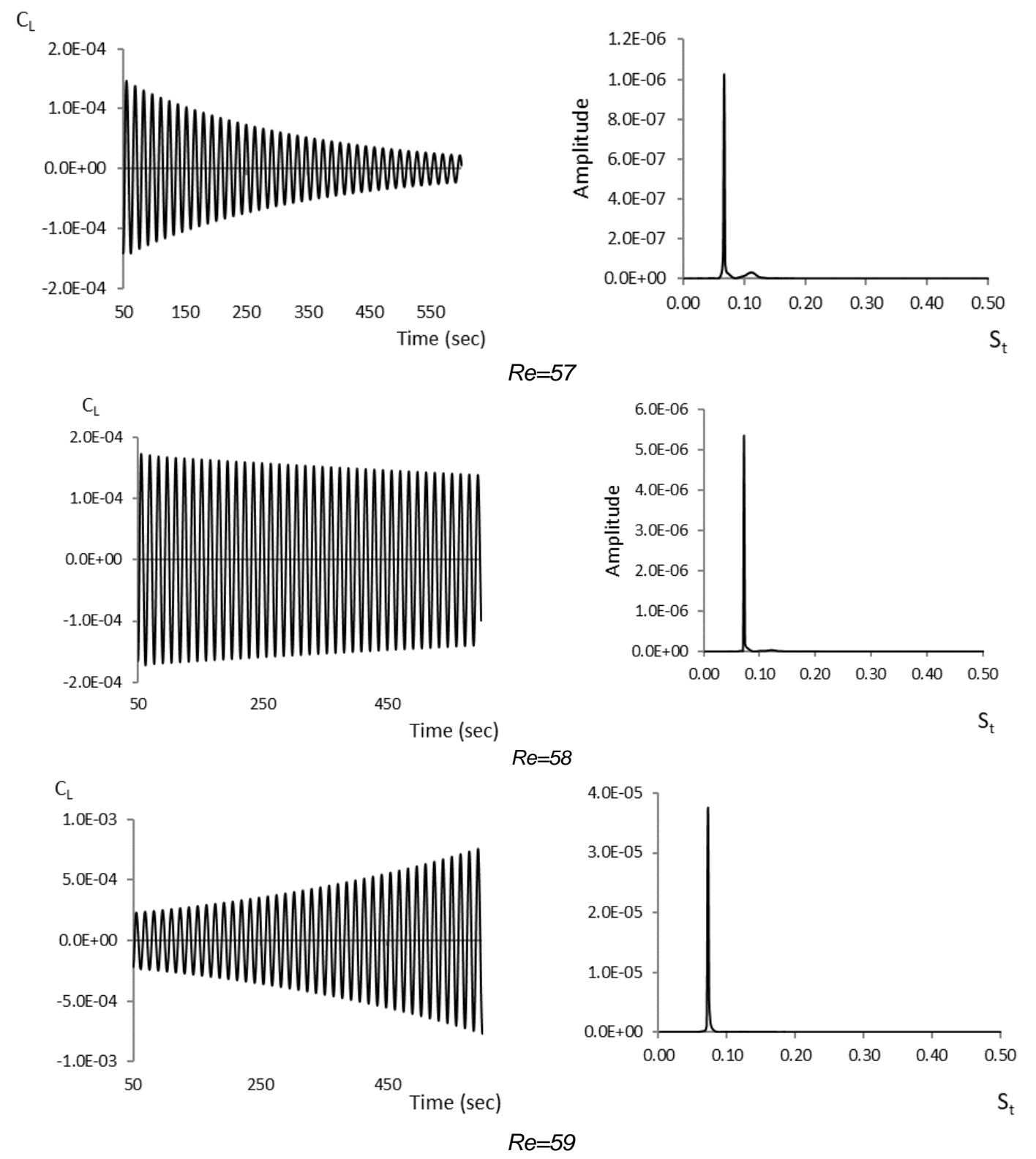

Figure 13: Lift time history and amplitude of textured model at $R e=57,58$ and 59.

Contours of the velocity in $z$ direction of the textured pipe are depicted in Fig. 14 and show disorganised flow regime in the wake of the textured facets. It is evident from the figure that a fluctuating downward flow is generated at the onset of negative lift, in the wake region of the pipeline at the middle section with a length of 7D. It is meaningful to compare the flow profiles of the circular and textured cylinders in the cross-sectional planes. The evolution of the streamwise velocity in the yz planes at several downstream locations at mid-height of the two cylinders, at the onset of negative lift with $\mathrm{Re}=100$ are presented in Fig. 15. The cylinders are centred at $X / D=Y / D=15$ within the 3D-mesh. As shown in the figure, pairs of thin shear layers are generated in the immediate wakes of the cylinders. Shear layers of the textured pipe exhibit periodic undulations due to its wavy geometry which is felt most at $X / D=17$. The shear layers grow thicker further downstream and the undulations gradually diminish as is shown at $X / D=18$. It is also evident that the width of the wake in the span-wise direction of the textured pipe, indicated by the width between the two shear layers, varies with the downstream locations. At 
$\mathrm{X} / \mathrm{D}=17$ in the downstream of the textured pipe, the wake width at node (see Fig. 14) is slightly wider than that at the saddle.

The pressure and friction distributions on the surfaces of the node and saddle sections (highlighted in Fig. 14) at $\mathrm{Re}=100$ are presented in Fig. 16 at the onset of maximum negative lift force. As seen the stagnation and base pressures at the node and saddle are the same. The separation angle is 45 degrees which is the average of 30 and 60 degree orientations that denote the sharp upstream edges of the corner and face oriented hexagons associated with saddle and node sections respectively. The pressure and friction distributions of the node and saddle vary for $45<\theta<100$ and $260<\theta<315 \circ$ in the upstream surfaces. Local drag and lift coefficients at node and saddle are calculated by integrating pressure and shear stresses on the surfaces of the node and saddle in the in-line and cross-flow directions. The results are presented in Table 3 and show that the local drag at saddle is 7 percent lower than that of the node. The local lift at the node is however 11 percent larger than corresponding value at the saddle. These numbers agree with the results published in (Ahmed and Bays-Muchmore 1992; Nguyen and Jee 2004).

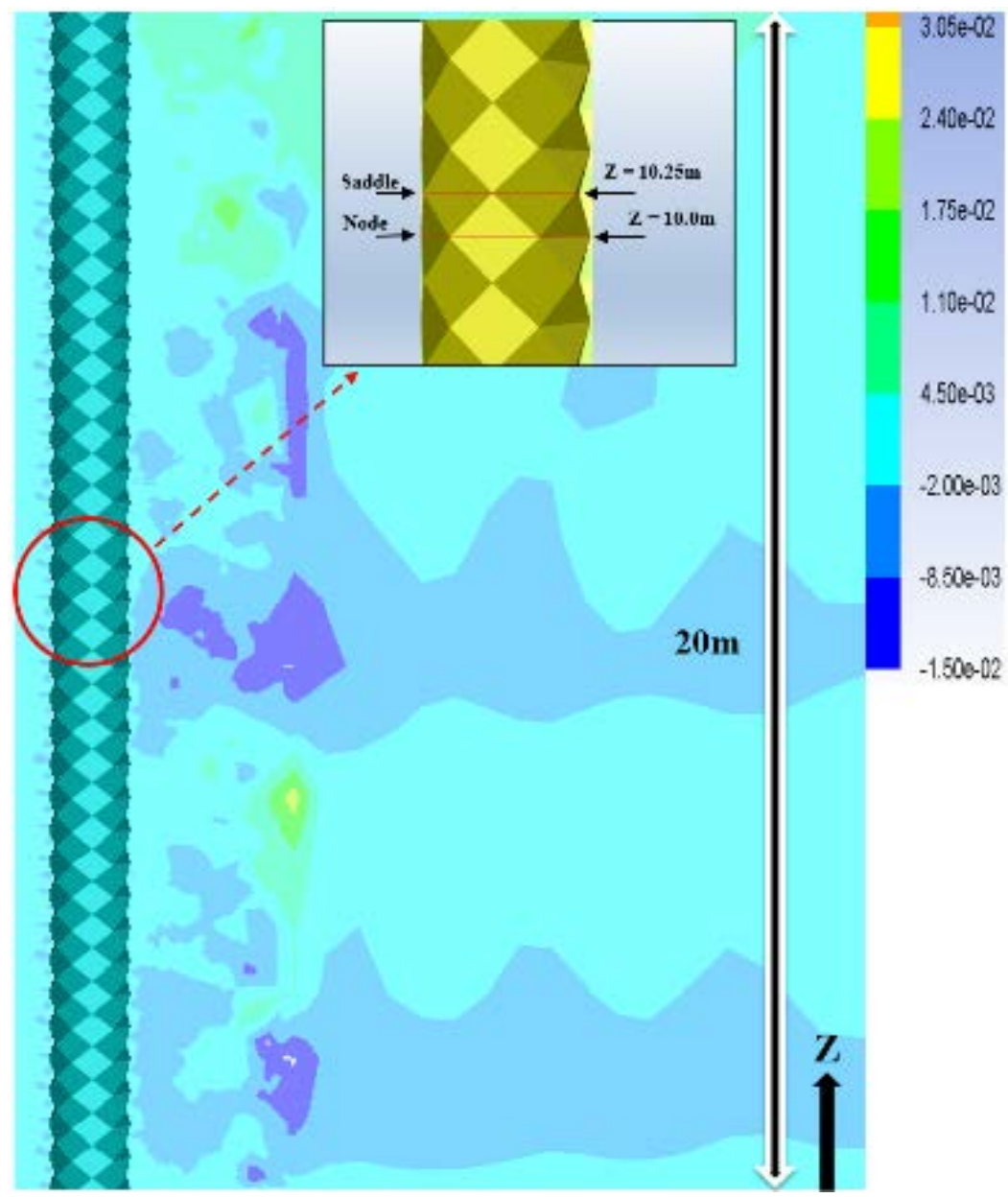

Figure 14: Contours of velocity $(\mathrm{m} / \mathrm{s})$ in the $z$ direction of the textured pipe at the onset of negative lift at $\mathrm{Re}=100$. 

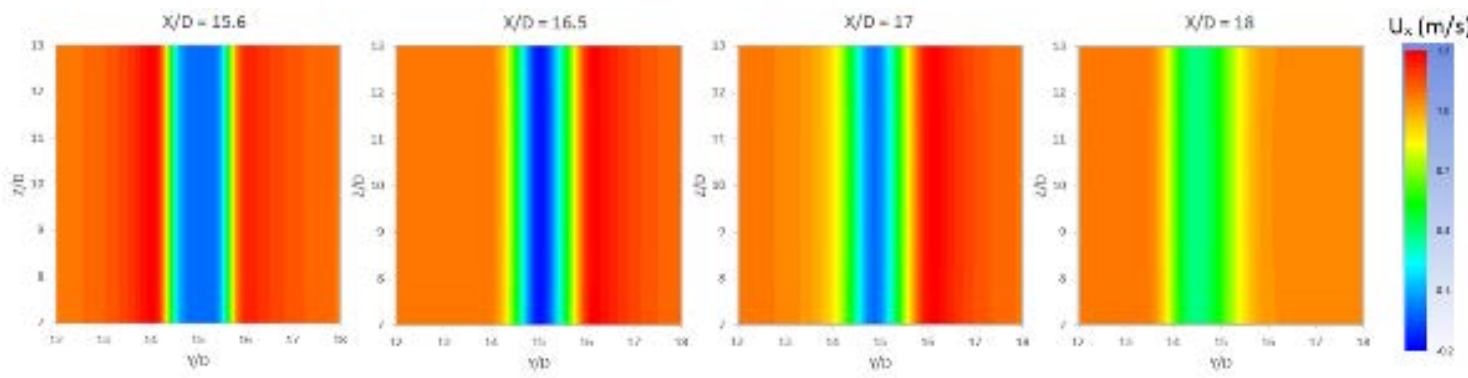

(a) Circular cylinder
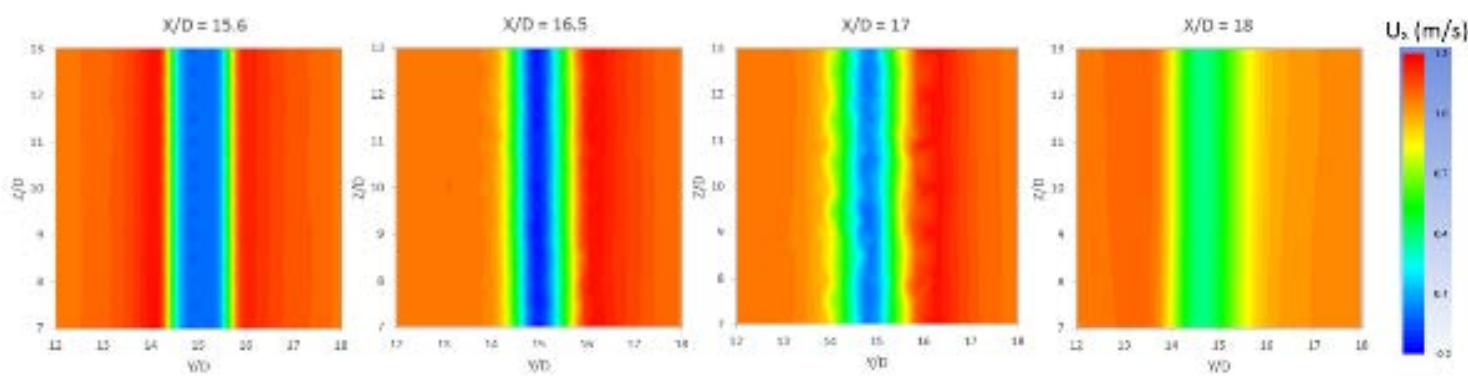

(b) Textured cylinder

Figure 15: Stream-wise velocity in the yz planes for the 3-dimenshional circular and textured cylinders at the onset of negative lift.

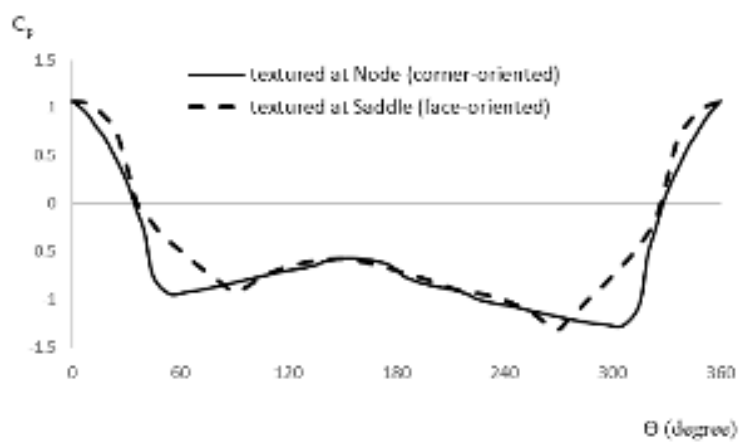

(a)

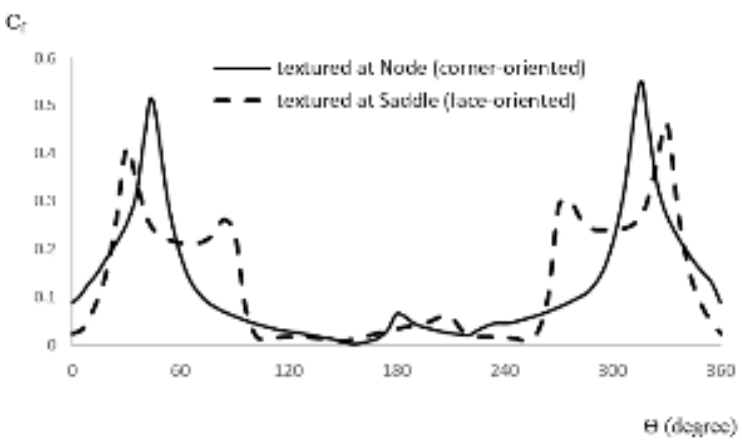

(b)

Figure 16: (a) Pressure and (b) skin friction distributions on the surface of the textured pipe at the onset of maximum negative lift force at node $(z=10.0 \mathrm{~m})$ and saddle $(z=10.25 \mathrm{~m})$ sections shown in Fig. 14 at $\mathrm{Re}=100$. 


\section{Conclusions}

In this study, the flow regime around hexagonal cylinders at two orientations namely; corneroriented and face-oriented were numerically investigated. Navier-Stokes and mass conservation (continuity) equations were solved using semi-implicit SIMPLE algorithm in 2D transient analysis. Characteristics of the in-line and cross-flow forces and vortex regimes were determined in terms of non-dimensional parameters, drag coefficient, lift coefficient and Strouhal number. Distributions of friction coefficients and pressure on the surface of the hexagons were discussed and streamline patterns and vorticities were depicted. Flow regime around a novel textured geometry was investigated using 3D transient analysis. The following conclusions can be deducted from the present study:

- The critical Reynolds number of the textured geometry is 58 which is higher than the $\mathrm{Rec}=52$ and 54 for the face-oriented and corner-oriented hexagons respectively.

- The drag coefficient of the corner-oriented hexagon is greater than the CD of the faceoriented hexagon for $\mathrm{Re}<200$. The drag coefficient of the textured geometry is in between those of the face-oriented and corner-oriented hexagons for a $\mathrm{Re}<140$ and smaller than both for $140<\operatorname{Re}<200$.

- The lift coefficient of the corner-oriented hexagon is smaller than the CL of the faceoriented hexagon for almost the entire Re range studied. Interestingly, the lift coefficient of the textured cylinder is smaller than the face-oriented and corner-oriented hexagons.

- The Strouhal number of the face-oriented hexagon is dominantly greater than those of the textured cylinder and corner-oriented hexagon.

- The Peak pressure coefficients of the corner-oriented and the face-oriented hexagons are close at $R e=200$, but the peak friction coefficient of the corner-oriented hexagon is substantially greater than that of the face-oriented hexagon at $R e=100$ and 200 .

- In both face-oriented and corner-oriented hexagons, the vortices at $\mathrm{Re}=200$ are centred closer to the cylinder wall compared to those at $\mathrm{Re}=100$.

- The results suggest that within the studied Reynolds number range i.e. $R e<200$, the drag coefficient of the textured geometry is almost equal to that of the circular cylinder while the lift coefficient of textured geometry is substantially smaller than that of circular, face-oriented and corner-oriented hexagon pipes.

- Local drag of the textured pipe at saddle is 7 percent lower than that of the node and the local lift at the node is 11 percent larger than corresponding value at the saddle.

\section{Acknowledgements}

The authors are grateful for SEED funding financial support from Griffith School of Engineering. We gratefully acknowledge the support of the Griffith University eResearch Services Team and the use of the High Performance Computing Cluster "Gowonda" to complete this research. 


\section{References}

Ahmed A, Bays-Muchmore B. 1992. Transverse flow over a wavy cylinder. Physics of Fluids A: Fluid Dynamics.4:1959-1967

Albermani F, Khalilpasha H, Karampour H. 2011. Propagation buckling in deep sub-sea pipelines. Engineering Structures. 9//;33:2547-2553.

ANSYS 16.1 Release Al. 275 Technology Drive, Canonsburg,PA 15317.

Baldock M, Karampour H, Sleep R, Vyltla A, Albermani F, Golshani A, Callaghan D, Roff G, Mumby $P, 2014$. Resilience of branching and massive corals to wave loading under sea level rise - A coupled computational fluid dynamics-structural analysis. Marine Pollution Bulletin,86: 91-101.

Bearman P, Zdravkovich M. 1978. Flow around a circular cylinder near a plane boundary. Journal of Fluid Mechanics.89:33-47.

Berthelsen PA, Faltinsen OM. 2008. A local directional ghost cell approach for incompressible viscous flow problems with irregular boundaries. J Comput Phys.227:4354-4397.

Braza M, Chassaing P, Minh HH. 1986. Numerical study and physical analysis of the pressure and velocity fields in the near wake of a circular cylinder. Journal of fluid mechanics.165:79130.

Dimopoulos HG, Hanratty TJ. 1968. Velocity gradients at the wall for flow around a cylinder for Reynolds numbers between 60 and 360. Journal of Fluid Mechanics.33:303-319.

Gallardo D, Sahni O, Bevilacqua R. 2017. Hammerstein-Wiener based reduced-order model for vortex-induced non-linear fluid-structure interaction. Engineering with Computers.33:219237.

Homann F. 1936. Einfluß großer Zähigkeit bei Strömung um Zylinder. Forschung auf dem Gebiete des Ingenieurwesens.7:1-10.

Karampour H, Alrsai M, Albermani F, Guan H, Jeng D. 2017. Propagation buckling in subsea Pipe-in-pipe systems. Journal of Engineering Mechanics. 143(9), 04017113

Karampour H, Albermani F. 2014. Experimental and numerical investigations of buckle interaction in subsea pipelines. Engineering Structures.66:81-88.

Karampour H, Albermani F. 2015. Buckle interaction in textured deep subsea pipelines. Ships and Offshore Structures.1-11.

Khaledi HA, Andersson HI. 2011. On vortex shedding from a hexagonal cylinder. Physics Letters A.375:4007-4021.

Kumar B, Mittal S. 2006. Prediction of the critical Reynolds number for flow past a circular cylinder. Computer Methods in Applied Mechanics and Engineering.195:6046-6058.

Lam K, Wang F, Li J, So R. 2004. Experimental investigation of the mean and fluctuating forces of wavy (varicose) cylinders in a cross-flow. Journal of fluids and structures.19:321-334.

Linnick MN, Fasel HF. 2005. A high-order immersed interface method for simulating unsteady incompressible flows on irregular domains. J Comput Phys.204:157-192.

Meliga P, Cadot O, Serre E. 2016. Experimental and Theoretical Sensitivity Analysis of Turbulent Flow Past a Square Cylinder. Flow, Turbulence and Combustion.97:987-1015.

Nguyen A, Jee S. Experimental investigation on wake behind a sinusoidal cylinder. Proceedings of the Proceeding of Tenth Asian Congress of Fluid Mechanics; 2004.

Norberg C. 1993. Flow around rectangular cylinders: pressure forces and wake frequencies. Journal of wind engineering and industrial aerodynamics.49:187-196.

Norberg C. 1994. An experimental investigation of the flow around a circular cylinder: influence of aspect ratio. Journal of Fluid Mechanics.258:287-316.

Okajima A, Yi DL, Sakuda A, Nakano T. 1997. Numerical study of blockage effects on aerodynamic characteristics of an oscillating rectangular cylinder. Journal of wind engineering and industrial aerodynamics.67-8:91-102.

Palei V, Seifert A. 2007. Effects of Periodic Excitation on the Flow Around a D-Shaped Cylinder at Low Reynolds Numbers. Flow, Turbulence and Combustion.78:409-428. 
Park J, Kwon K, Choi H. 1998. Numerical solutions of flow past a circular cylinder at Reynolds numbers up to 160. KSME international Journal.12:1200-1205.

Rajani BN, Kandasamy A, Majumdar S. 2009. Numerical simulation of laminar flow past a circular cylinder. Applied Mathematical Modelling.33:1228-1247.

Singh SP, Biswas G. 2013. Vortex induced vibrations of a square cylinder at subcritical Reynolds numbers. Journal of Fluids and Structures. 8//;41:146-155.

Sohankar A, Norberg C, Davidson L. 1998a. Low-Reynolds-number flow around a square cylinder at incidence: Study of blockage, onset of vortex shedding and outlet boundary condition. International Journal for Numerical Methods in Fluids.26:39-56.

Sohankar A, Norberg C, Davidson L. 1998b. Low-Reynolds-number flow around a square cylinder at incidence: study of blockage, onset of vortex shedding and outlet boundary condition. International journal for numerical methods in fluids.26:39-56.

Sparrow EM, Abraham JP, Tong JCK. 2004. Archival correlations for average heat transfer coefficients for non-circular and circular cylinders and for spheres in cross-flow. International Journal of Heat and Mass Transfer. 11//;47:5285-5296.

Stephan P, Love C, Albermani F, Karampour H. 2015. Experimental study on confined buckle propagation. Advances in structural engineering.

Sumer BM, Fredsøe J. 1997. Hydrodynamics around cylindrical structures River Edge, N.J;London;Singapore;: World Scientific.

Sumner D, Akosile O. 2003. On uniform planar shear flow around a circular cylinder at subcritical Reynolds number. Journal of Fluids and Structures.18:441-454.

Swaddiwudhipong S, Anh TTT, Liu ZS, Hua J. 2007. Modelling of wind load on single and staggered dual buildings. Engineering with Computers.23:215-227.

Thom A. 1933. The Flow Past Circular Cylinders at Low Speeds. Proceedings of the Royal Society of London A: Mathematical, Physical and Engineering Sciences. 1933-09-01 00:00:00;141:651-669.

Tian ZW, Wu ZN. 2009. A study of two-dimensional flow past regular polygons via conformal mapping. Journal of Fluid Mechanics.628:121-154.

Travin A, Shur M, Strelets M, Spalart P. 2000. Detached-Eddy Simulations Past a Circular Cylinder. Flow, Turbulence and Combustion.63:293-313.

Williamson C. 1988. The existence of two stages in the transition to three-dimensionality of a cylinder wake. The Physics of fluids.31:3165-3168.

Williamson C. 1989. Oblique and parallel modes of vortex shedding in the wake of a circular cylinder at low Reynolds numbers. Journal of Fluid Mechanics.206:579-627.

Williamson C. 1996. Three-dimensional wake transition. In: Advances in Turbulence VI. Springer. p. 399-402.

Williamson CHK, Roshko A. 1988. Vortex formation in the wake of an oscillating cylinder. Journal of Fluids and Structures. 7//;2:355-381. Wood JN, De Nayer G, Schmidt S, Breuer M. 2016. Experimental Investigation and Large-Eddy Simulation of the Turbulent Flow past a Smooth and Rigid Hemisphere. Flow, Turbulence and Combustion.97:79-119.

Yang K-S, Choi C-B, Yoon D-H. 2010. Flow past a square cylinder with an angle of incidence. Physics of Fluids.22:043603-043603-043612.

Zdravkovich MM. 2003. Flow around Circular Cylinders: Volume 2: Applications: Oxford university press. 